\title{
Der Einfluss von Koalitionsaussagen, programmatischen Standpunkten und der Bundespolitik auf die Regierungsbildung in den deutschen Ländern
}

\author{
Thomas Bräuninger · Marc Debus
}

Zusammenfassung: Der parteipolitischen Zusammensetzung der Landesregierungen kommt im deutschen Mehrebenensystem eine wichtige Bedeutung zu. Dies gilt nicht nur im Hinblick auf die gesetzgeberische Tätigkeit des Bundes, sondern auch für die Muster und die Entwicklung des Parteienwettbewerbs auf Landes- und Bundesebene. In diesem Aufsatz wird der Frage nachgegangen, welche in der international-vergleichenden Literatur zur Koalitionsbildung gängigen und welche für das deutsche Mehrebenensystem zusätzlich relevanten Faktoren einen Einfluss auf die Regierungsbildung in den Bundesländern haben. Die empirische Untersuchung für den Zeitraum ab der deutschen Wiedervereinigung stützt sich auf originäre Informationen über die bundespolitischen Rahmenbedingungen sowie die Koalitionsaussagen und die programmatischen Positionen der Landesparteien. Die Ergebnisse zeigen, dass die programmatischen Haltungen der Landesparteien neben Faktoren wie der Stärke der Parteien im Parlament, den vor der Wahl getätigten Koalitionsaussagen und der parteipolitischen Zusammensetzung von Regierung und Opposition auf Bundesebene einen wesentlichen Einfluss auf die Koalitionsbildung in den Bundesländern haben.

Schlagwörter: Koalitionsbildung • Parteienwettbewerb - Mehrebenensystem • Deutsche Bundesländer $\cdot$ Wahlprogrammanalyse

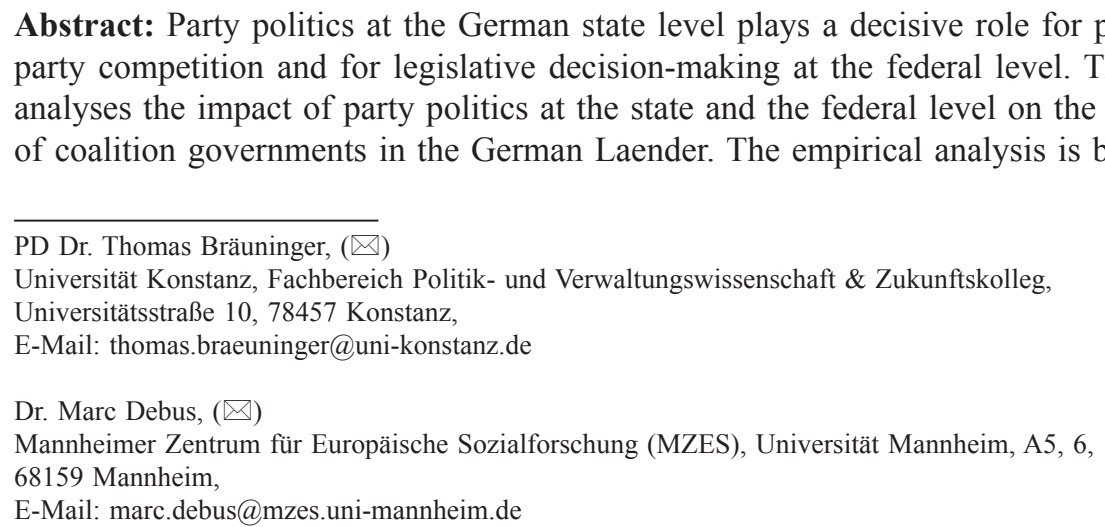


unique dataset that covers information on the state parties' programmatic positions, their pre-electoral alliances, and the structure of party competition on the federal level in the time period between 1990 and 2007. The results reveal that the programmatic positions of state parties have a decisive impact on government formation. Other relevant factors are the parties' relative strength, their coalition preferences and the partisan composition of government and opposition on the federal level.

Keywords: Coalition Formation $\cdot$ Party Competition $\cdot$ Multi-level Settings $\cdot$ German Laender $\cdot$ Analysis of Election Manifestos

\section{Einleitung und Fragestellung}

Der parteipolitischen Zusammensetzung der Regierungen in den deutschen Bundesländern kommt aufgrund der potenziellen Vetospielerfunktion der jeweiligen Parteien im Bundesrat eine große bundespolitische Bedeutung zu. So bedurften bis zum Inkrafttreten der Föderalismusreform 2006 mehr als die Hälfte der Gesetzesinitiativen der Zustimmung des Bundesrates, und dieser Anteil wird auch nach der Reform nicht wesentlich unter 40 Prozent sinken (Deutscher Bundestag 2006: 14-15; Schmidt 2007: 203-204, 218). Entsprechend ist insbesondere die jeweilige Führung der Bundesparteien nach wie vor ausnehmend an Regierungskoalitionen auf Landesebene interessiert, die parteipolitisch mit dem Regierungs- bzw. Oppositionslager im Bundestag kongruent sind. Allerdings führen Regierungsbildungsprozesse in den Bundesländern immer wieder zu Koalitionen, welche die parteipolitische Lagerbildung von Regierung und Opposition auf Bundesebene durchbrechen. Dies war jüngst in Bremen nach den Bürgerschaftswahlen im Mai 2007 der Fall. Dort löste ein Bündnis aus Sozialdemokraten (SPD) und Grünen die seit 1995 bestehende Große Koalition von SPD und Christdemokraten (CDU) ab. Dies ist umso erstaunlicher, da eine Fortführung der SPD/CDU-Koalition nicht nur mit der bundespolitischen Konstellation übereingestimmt hätte. Eine rot-schwarze Koalition in Bremen hätte auch die für Grundgesetzänderungen wichtige Zweidrittelmehrheit der Berliner Großen Koalition im Bundesrat weiterhin gesichert. Eine mögliche Erklärung für das Ende der Koalition aus Union und SPD ist, dass sich die beiden Landesorganisationen von Sozial- und Christdemokraten im Zeitverlauf programmatisch voneinander entfernt und der Bremer SPD eine rot-grüne Koalition aus sachpolitisch-inhaltlichen Gründen nun näher liegender erschien. Auch deutete die während des Wahlkampfs seitens der SPD geäußerte Koalitionspräferenz bereits darauf hin, dass eine Fortsetzung der großen Koalition einem rot-grünen Bündnis zumindest nicht vorgezogen wurde (vgl. Frankfurter Allgemeine Zeitung vom 12. Mai 2007: 4).

Das Bremer Beispiel macht deutlich, dass das Verhalten der landespolitischen Akteure von unterschiedlichen Zielen motiviert sein kann, deren Erreichung sich oftmals ausschließt. Es mag bei der Bremer SPD-Entscheidung für Rot-Grün um die Aussicht der Verwirklichung spezifischer landespolitischer Ziele gegangen sein oder auch um den relativen Vorteil bei der Besetzung von Regierungsämtern in einer Koalition mit kleinem Partner. In anderen Fällen kann die Aussicht, politische Inhalte auf der bundespolitischen Ebene mittels des Bundesrats durchsetzen oder auch verhindern zu können, eine 
Rolle spielen. In diesem Fall läge die Bildung von (Koalitions-)Regierungen nahe, die mit dem Regierungs- bzw. Oppositionslager auf Bundesebene übereinstimmen. Und schließlich mögen aus genau diesem Grund auch bundespolitische Akteure einen Anreiz haben, Einfluss auf die Regierungsbildung in den Ländern zu nehmen. Sind die Landesregierungen im Amt, setzt sich der Zielkonflikt auf der Ebene des Bundesrates fort, wenn landes- oder landesparteipolitische Interessen den wahlstrategischen Überlegungen der zugehörigen Bundesparteien entgegenstehen (Lehmbruch 1976; Scharpf 1989; Zohlnhöfer 1999).

Angesichts der Diskussion um „Blockadepolitik“ und „Reformstau“ im deutschen Föderalismus wurde die Frage nach dem Einfluss der Bundespolitik auf das (Bundesrats-)Verhalten der Landesregierungen in der politikwissenschaftlichen Literatur eingehend behandelt (z. B. Scharpf 2000; König 2001; Bräuninger/Ganghof 2005). Weniger systematisch wurde hingegen die Frage nach der Bedeutung der Bundespolitik bei der Bildung von Landesregierungen thematisiert (vgl. jedoch Fabritius 1976; Lehmbruch 1976; Jun 1994; Downs 1998: 106-120; Kropp 2001; Pappi et al. 2005). Ein Grund hierfür mag sein, dass das Argument der bundespolitischen Einflussnahme voraussetzt, dass bundes- und landespolitische Interessen innerhalb einer Partei divergieren, der Nachweis dieser Unterschiede jedoch schwer zu führen ist. Falls etwa in Bremen im Mai 2007 wiederum eine SPD/CDU-Regierung gebildet worden wäre, dann könnte ein bundespolitischer Einfluss höchstens dann vorliegen, wenn die programmatische Nähe der beiden Parteien nicht allenthalben für eine Große Koalition gesprochen hätte. Da die programmatische Konfliktlage sich auf der Bundesebene unter Umständen anders als auf der Landesebene darstellt, kann eine derartige Analyse nicht ohne die Kenntnis der programmatischen Ausrichtung der Landesverbände der Parteien in den jeweiligen Ländern auskommen.

Der vorliegende Beitrag widmet sich diesem Themenkomplex und untersucht, welche Faktoren die Koalitionsbildung in den deutschen Ländern beeinflussen. Dazu ziehen wir Informationen über die inhaltlich-programmatische Positionierung der Landesparteien in den Bundesländern zwischen 1990 und 2007 heran. ${ }^{1}$ Das Hauptaugenmerk liegt dabei auf der Frage, welche Bedeutung bei der Koalitionsbildung dem bundespolitischen Kalkül im Vergleich zu landespolitischen Faktoren zukommt. Die Berücksichtigung der Positionierung der Landesparteien im Politikraum erlaubt damit nach unserem Kenntnisstand zum ersten Mal, die relative Bedeutung der beiden Faktoren in einer quantitativ-vergleichenden Untersuchung abzuschätzen und zu bewerten.

Dabei wird folgendermaßen vorgegangen: Das nächste Kapitel gibt zunächst einen Überblick über mögliche Faktoren, welche die Koalitionsbildung, insbesondere im Hinblick auf das Regieren im deutschen Mehrebenensystem, beeinflussen könnten. In Kapitel 3 werden dann der hier verwendete Datensatz sowie das statistische Analyseverfahren vorgestellt. Im Anschluss daran verdeutlichen wir den eigenständigen Charakter der programmatischen Positionierung von Landesparteien am Beispiel zweier Bundesländer. Das vierte Kapitel stellt die Ergebnisse der Untersuchung vor. Die Schlussbe-

1 Wir folgen einer üblichen und sprachvereinfachenden Konvention im Bereich der Koalitionsforschung und subsumieren auch die Bildung von Einparteiregierungen unter den Begriff der Koalitionsbildung. 
trachtung fasst die gewonnenen Erkenntnisse zusammen und diskutiert Anknüpfungspunkte für weitere Studien.

\section{Theoretische Ansätze zur Erklärung der Koalitionsbildung}

Moderne repräsentative Demokratien sind durch Akte der Delegation gekennzeichnet, mit denen die politischen Zielvorstellungen der Wähler als ultimativen Prinzipalen durch die Übertragung von Entscheidungskompetenz an Abgeordnete, Regierungen, Minister und Verwaltungen ihren Ausdruck finden. In diesem Prozess stellt die Bildung von Regierungen in parlamentarischen Systemen nur ein Glied einer langen Kette von Delegationen dar. Einerseits sind Regierungsbildungen das Ergebnis der Verhandlungen von - in der Regel parteilichen - politischen Akteuren, deren Verhandlungsmandat sich in letzter Konsequenz von der Wählerunterstützung herleitet. Andererseits bilden sie den Ausgangspunkt für das Regierungshandeln, das als output im Sinne Eastons (1965) die Unterstützung und letztlich die Wiederwahlchancen der Regierung(sparteien) bestimmt. Will man den Prozess der Regierungsbildung verstehen, dann kann dies schwerlich glücken, wenn nicht dieser dynamische Zusammenhang der Delegation von Handlungskompetenzen in den Blick genommen wird (Austen-Smith/Banks 1988; Diermeier et al. 2003). Entsprechend hat die Forschung in diesem Bereich zunehmend der Abhängigkeit der eigentlichen Koalitionsbildung von mehreren Faktoren Rechnung getragen: einerseits der Abhängigkeit von den Randbedingungen der Regierungsbildung, etwa dem Wählerverhalten und dem strategischen Parteienwettbewerb, andererseits aber auch den Erwartungen von Wählern und politischen Akteuren an die zukünftige Performanz von Regierungen.

Abbildung 1 veranschaulicht schematisch diese Verknüpfung der Koalitionstheorie mit zentralen Fragen der Wähler- und Parteienforschung, wobei wir zwischen drei Typen von Akteuren unterscheiden wollen. Dies sind erstens die Wähler, die den output von bestehenden Regierungen (zum Zeitpunkt t-1) bewerten, Unterstützung für politische Parteien leisten und mit ihrer Wahlentscheidung am Ende der Legislaturperiode die parlamentarischen Mehrheitsverhältnisse bestimmen, welche den Ausgangspunkt für die neuerliche Regierungsbildung (zum Zeitpunkt t) darstellen. Zweitens stellen die Landesparteien relevante Akteure dar, die mit bestimmten Positionen zu Sachfragen um Wählerstimmen in Wettbewerb zueinander treten, deren sachpolitische Positionierung allerdings die Möglichkeit und Attraktivität bestimmter Koalitionen festlegt und die sich entsprechend bereits vor der Wahl für Koalitionsoptionen aussprechen bzw. Vorwahlallianzen eingehen. Drittens kommt im föderalen Mehrebenensystem den Bundesparteien eine entscheidende Rolle $\mathrm{zu}$, da sie aus bundespolitischen Überlegungen, z.B. Stärkung des eigenen Lagers im Bundesrat oder Vorbildfunktion landespolitischer Koalitionen, die Bildung bestimmter Koalitionen auf Landesebene befürworten oder ablehnen können.

Die Abbildung deutet die Vielzahl der Faktoren an, die bei der Bildung einer Regierung letztlich eine Rolle spielen können, etwa die Erwartungen der Parteien bezüglich des langfristigen output einer Koalition (z. B. Austen-Smith/Banks 1988), die Berücksichtigung der Koalitionspräferenzen der Parteien durch die Wähler in Form strategischen 


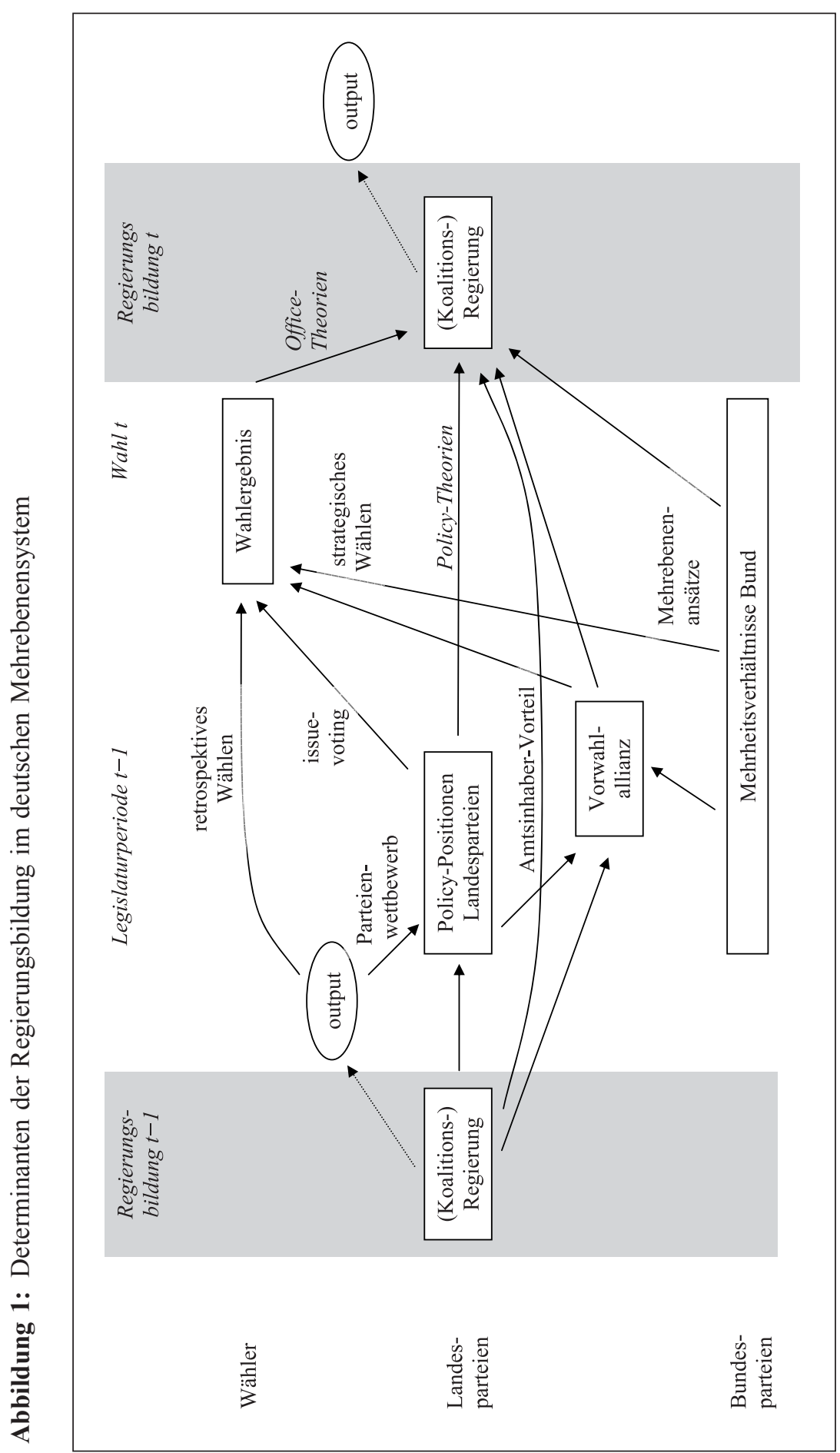


Koalitionswählens (z. B. Pappi/Gschwend 2005) oder das Einkalkulieren der bundespolitischen Implikationen einer Landtagswahl (z. B. Dinkel 1977). Wir werden unsere folgende Untersuchung auf die vier Faktoren bzw. Prozesse beschränken, die der Regierungsbildung unmittelbar vorgelagert sind: das numerische Wahlergebnis bzw. die Sitzstärke der Parteien, die sachpolitische Positionierung der Parteien, den bundes- und landespolitischen Kontext und die eventuell geäußerten Vorwahlallianzen von Parteien. Im Falle der Vorwahlallianzen wollen wir explizit berücksichtigen, dass diese nicht unabhängig von den restlichen Faktoren geschlossen werden; Vorwahlallianzen sind zukünftige Regierungen in statu nascendi und sollten deshalb in ähnlicher Weise von denselben Faktoren abhängen. Um zutreffende Aussagen über einzelne Einflussgrößen machen zu können, werden wir diese Endogenität der Vorwahlallianz berücksichtigen müssen. Es ließe sich gleichsam argumentieren, die sachpolitische Positionierung der Parteien, die Entscheidung der Wähler oder der bundespolitische Kontext und die Wählerentscheidung seien letztlich keine unabhängigen Faktoren. Wir sehen allerdings von einer expliziten Modellierung dieser Zusammenhänge ab, da wir davon ausgehen können, dass sie - zumindest im Vergleich zur Vorwahlallianz - von eher schwacher Ausprägung sind.

- Numerisches Wahlergebnis: Die Anzahl und die Sitzstärke der im Parlament vertretenen Parteien bestimmen zunächst einmal die Menge der möglichen Koalitionen, die eine mehrheitsfähige Regierung bilden könnten. Wenn die Parteien lediglich an Ämtern interessiert sind, sind Sitzstärke und Anzahl der Koalitionspartner von Interesse. Entsprechend gehen sogenannte office-orientierte Ansätze der (kooperativen) Koalitionstheorie davon aus, dass sich minimale Gewinnkoalitionen (von Neumann/ Morgenstern 1944) bzw. Gewinnkoalitionen mit einer möglichst geringen Anzahl an Parteien bilden, sodass Verhandlungskosten gering ausfallen (Leiserson 1968). Auch in nicht-kooperativen Ansätzen kommt der Sitzstärke der Parteien eine wichtige Bedeutung zu - sowohl bei der Wahl des „Formateurs“ oder Regierungsbildners als auch bei den Koalitionsverhandlungen. So betonen Austen-Smith und Banks (1988) die Verhandlungsmacht der sitzstärksten Partei, die beispielsweise in einem aus drei Fraktionen bestehenden Parlament ein Bündnis mit der kleinsten Partei bilden kann (ähnlich Baron/Ferejohn 1989; Baron/Diermeier 2001). ${ }^{2}$

- Sachpolitische Parteipositionen: Unabhängig von der Zugehörigkeit zu parteipolitischen Lagern nehmen Landesparteien programmatische Standpunkte zu sachpolitischen Fragen ein. Diese Positionen bilden sowohl die Grundlage für das issuevoting der Wähler als auch für die Koalitionspräferenzen von sachpolitisch-motivierten Parteien, die Koalitionen anhand ihres zu erwartenden output bewerten. Im Allge-

2 Wir gehen an dieser Stelle nicht weiter auf die Literatur ein, welche die Rolle des „Formateurs" in den Vordergrund rückt, da es auf der Ebene der deutschen Bundesländer kein Staatsoberhaupt gibt, das einen politischen Akteur mit der Regierungsbildung beauftragt (vgl. für solche Ansätze etwa Warwick 1996; Diermeier et al. 2003). Letztgenanntes gilt im Übrigen auch auf Bundesebene: Der Bundespräsident ernennt zwar den Bundeskanzler, erteilt jedoch keinen Auftrag zur Regierungsbildung (vgl. Schmidt 2007: 176). Die Vorverhandlungen und Sondierungsgespräche zur Regierungsbildung werden vielmehr von den Vertretern der im Parlament vertretenen Parteien selbst organisiert. 
meinen wird eine Partei Koalitionen anstreben, deren erwartbare Politik der eigenen Position nahekommt. Daher beziehen policy-orientierte Ansätze der Koalitionstheorie die ideologischen bzw. politikfeldspezifischen Positionen der Parteien ein und prognostizieren die Wahl von Koalitionen mit geringer Heterogenität (vgl. Axelrod 1970). ${ }^{3}$ Koalitionsbildung ist aus der policy-orientierten Perspektive jedoch nicht nur abhängig von den programmatischen Positionen der Parteien. Ein weiterer Faktor ist das Gewicht, das die Parteien den jeweiligen Politikfeldern zuweisen (Hinich/ Munger 1997: 77-82). So ist anzunehmen, dass beispielsweise für eine wirtschaftsliberale Partei wie die FDP das sozioökonomische Politikfeld von größerer Bedeutung ist als das gesellschaftspolitische, während für die gesellschaftspolitisch konservative Partei Bibeltreuer Christen (PBC) das letztgenannte Politikfeld eine vergleichsweise große Bedeutung hat. Dementsprechend sollte die FDP weniger kompromissbereit in wirtschafts- und sozialpolitischen und die PBC in gesellschaftspolitischen Fragen sein, was wiederum die wahrgenommene Distanz zur Position der anderen Parteien beeinflusst. ${ }^{4}$

Nun können sich aber die strukturellen Rahmenbedingungen innerhalb von Mehrebenensystemen deutlich unterscheiden (vgl. Deschouwer 2003). So sollten landesspezifische politisch-kulturelle wie sozio-ökonomische Faktoren einen Einfluss auf die ideologisch-programmatische Positionierung der Parteien ausüben. Dazu zählen in Deutschland vor allem langfristig ausgerichtete Determinanten wie die Wirtschafts- oder Konfessionsstruktur (vgl. Rohe 1992; Wehling 2006; Flick/Vatter 2007: 46-48; Freitag/Vatter 2008). Beispielsweise charakterisieren von Alemann und Brandenburg (2000: 124-126) die nordrhein-westfälische SPD als wenig ideologisierte und gewerkschaftsnahe „Traditionstruppe“, die stark auf die Arbeitnehmerinteressen in diesem industriell geprägten Bundesland eingeht und daher gesellschaftsoder umweltpolitisch progressiven Forderungen eher skeptisch gegenübersteht. Schmid (1990: 75-120) argumentiert, dass die Union in stark vom katholischen „sozial-moralischen Milieu“ (Lepsius 1973) geprägten Landesverbänden der CDU, wie denen Nordrhein-Westfalens, Baden-Württembergs und des Saarlands, wirtschafts- und sozialpolitisch moderater eingestellt ist als in norddeutsch-protestantischen Bundesländern. Dass sich die programmatischen Ausrichtungen von Landesund Bundesparteien sowie innerhalb von CDU/CSU, SPD, FDP, Bündnis 90/Die

3 Für eine Anwendung des Modells von Sened (1995, 1996), welches office- und policy-orientierte Theorien der Koalitionsbildung kombiniert, auf die Regierungsbildung in den deutschen Bundesländern siehe Shikano/Linhart (2009).

4 In Anlehnung an die Bedeutung von Salienzen hat Warwick (2006) sogenannte Politikhorizonte (policy horizons) als einen weiteren relevanten Faktor der Koalitionsbildung vorgeschlagen. Diesem Ansatz zufolge akzeptieren Parteien nicht automatisch alle Politikvorschläge, die ihrem Idealpunkt näher kommen als der Status quo, wie dies in gängigen polit-ökonomischen Modellen der Fall ist (vgl. Laver/Shepsle 1996: 46-49; Hinich/Munger 1997: 52-64). Stattdessen weist jede Partei eine - nach Politikfeld variierende - begrenzte Bereitschaft auf, Kompromisse einzugehen. Die Grenze dieser Bereitschaft markiert Warwick (2006) zufolge den Politikhorizont jeder Partei. Für eine Anwendung dieses Konzepts auf die deutschen Landesparteien fehlen uns jedoch geeignete Daten. 
Grünen und $\mathrm{PDS}^{5}$ in der Tat voneinander unterscheiden, konnte bereits gezeigt werden (vgl. Debus 2007a; Bräuninger/Debus 2008). Daher erscheint das Heranziehen der programmatischen Positionen der Bundesparteien zur Analyse der Koalitionsbildung in den Bundesländern, wie es von Pappi, Becker und Herzog (2005) vorgeschlagen wurde, nicht ausreichend. Vielmehr müssen sowohl die programmatischen Positionen als auch die relative Bedeutung der Politikfelder für die jeweiligen Parteien auf der Ebene der Landesparteien bestimmt werden, um zu untersuchen, welchen Einfluss sachpolitische Faktoren auf die Koalitionsbildung in den Ländern haben.

- Bundespolitischer Kontext: Im föderalen Mehrebenensystem stehen allgemeine Wahlen auf bundes- und gliedstaatlicher Ebene selten unverbunden nebeneinander. So können Wähler, die etwa mit der Arbeit der Bundesregierung unzufrieden sind, die Wahlen zu den Landesparlamenten dazu nutzen, die im Bund regierenden Parteien abzustrafen, und so der bundespolitischen Opposition zu mehr Einfluss im Bundesrat verhelfen. Eine solche Form strategischen Wählens bei Landtagswahlen ist bereits mehrfach nachgewiesen worden (vgl. z. B. Dinkel 1977; Hough/Jeffery 2003; Burkhart 2005). Gleichzeitig wirken jedoch die bundespolitischen Mehrheitsverhältnisse auf die Regierungsbildung in den Bundesländern ein (vgl. Fabritius 1976; Lehmbruch 1976; Jun 1994; Kropp 2001; Pappi et al. 2005). Um ihre Politikziele auch im Bundesrat durchsetzen zu können, sollten die die Bundesregierung tragenden Parteien versuchen, parteipolitisch gleiche Landesregierungen zu installieren, um damit das Lager der sogenannten Regierungs- oder R-Länder im Bundesrat zu stärken. Zumindest aber wären solche Parteikonstellationen zu verhindern, die nur bundespolitische Oppositionsparteien beinhalten und die Gruppe der Oppositionsländer (O-Länder) vergrößerten. Umgekehrt ist zu erwarten, dass die Oppositionsparteien auf Bundesebene die Schwächung des Regierungslagers (R-Länder) zum Ziel haben.

- Landespolitischer Kontext: Aus dem Blickwinkel der behaviouralistischen Schule der Politikwissenschaft kommt weniger den Office- und Policy-Faktoren als vielmehr kontextuellen Merkmalen ein entscheidender Einfluss auf die parteipolitische Zusammensetzung von Regierungen zu (vgl. Strøm 1990a; Strøm et al. 1994; Kropp et al. 2002: 26-37). Als landespolitischer Kontextfaktor kann der Amtsinhaberbonus gelten, der bestehenden Koalitionen zugutekommt - auch hier sowohl bei der Unterstützung durch Wähler als auch bei der Regierungsbildung. So weisen Koalitionsoptionen mit Parteien, mit denen in der Vergangenheit bereits Erfahrungen im gemeinsamen Regieren gesammelt werden konnten, im Allgemeinen geringere Unsicherheiten und geringere Transaktionskosten auf (vgl. Franklin/Mackie 1983; Bäck/Dumont 2007). Zu den Kontextmerkmalen zählt auch der Ausschluss von einer oder mehreren Parteien aus dem Koalitionsspiel aufgrund ihres Status als „AntiSystem-Partei“ (vgl. Powell 2000). Einzelne Parteien können zum einen generell,

5 Die PDS trat zur Bundestagswahl 2005 unter dem Namen „Linkspartei.PDS“ an und nahm nach der Fusion mit der „Wahlalternative Arbeit \& soziale Gerechtigkeit“ (WASG) den Namen DIE LINKE. an. Der Einfachheit halber verwenden wir im Folgenden auch für den Zeitraum seit 2005 die Abkürzung PDS. 
also von allen anderen Mitbewerbern, von der Regierungsbildung a priori ausgeschlossen werden, zum anderen aber auch nur von einigen Parteien als von vornherein „nicht regierungsfähig“ deklariert werden. Gleiches gilt - mit umgekehrtem Vorzeichen - für positiv formulierte Koalitionsaussagen oder Vorwahlallianzen. Solche werden in der Literatur zwar häufig im Hinblick auf das Wahlsystem im jeweiligen Land untersucht (Golder 2005; für Deutschland vgl. Pappi et al. 2006; Linhart 2007), vereinzelt auch hinsichtlich ihres Einflusses auf die Koalitionsbildung (vgl. Martin/Stevenson 2001; Debus 2007b; für die deutschen Bundesländer vgl. Jun 1994). Die letztgenannten Studien weisen auf den eigenständigen Effekt von vor der Wahl getroffenen Koalitionsaussagen auf das Ergebnis des Regierungsbildungsprozesses hin.

Wir wollen auch diesen Faktor in unserer Analyse berücksichtigen. Allerdings mahnt Abbildung 1 zur Vorsicht. Sie macht deutlich, dass die Bildung einer Vorwahlallianz von ähnlichen Faktoren abhängt wie die Bildung der Regierung nach der Wahl. Damit liegt auch bei weitgehender Kongruenz von Vorwahlallianz und Nachwahl-Koalition nicht notwendigerweise ein eigenständiger Einfluss der Vorwahlallianzen vor. Gleichzeitig würden wir den Stellenwert der programmatischen Positionen der beteiligten Parteien oder der bundespolitischen Konstellation unterschätzen, da diese Information bereits im Faktor Vorwahlallianz eingeschlossen ist. Bevor wir eine statistische Lösung für dieses Problem erläutern und die Ergebnisse präsentieren, soll zunächst auf die hier verwendeten Daten sowie die analytische Methode eingegangen werden.

\section{Daten und Methoden}

Zur Beantwortung der Fragestellung müssen drei Gruppen von unabhängigen Variablen identifiziert werden. Zunächst benötigen wir Informationen über die Anzahl sowie die relative Stärke der Parteien in den 16 Landesparlamenten von 1990 bis 2007. Diese Daten sind relativ leicht über gängige Datenhandbücher (vgl. Andersen/Woyke 2003) und etablierte Studien zum Parlamentarismus in den deutschen Bundesländern (vgl. Mielke/Reutter 2006) zugänglich. Auf dieser Grundlage kann für jede mögliche Parteienkonstellation bestimmt werden, ob sie die Charakteristika einer kleinen Gewinnkoalition (von Neumann/Morgenstern 1944) oder von Leisersons (1968) „,bargaining proposition“ aufweist. Des Weiteren liefern diese Daten Informationen darüber, ob die stärkste Partei im Parlament an den möglichen Koalitionen beteiligt ist (vgl. AustenSmith/Banks 1988) und ob die jeweilige Parteienkombination über eine Mehrheit im Parlament verfügt oder nicht (vgl. Strøm 1990b). Zweitens müssen Informationen über die Kontextfaktoren gesammelt werden, die für die Koalitionsbildung in den Bundesländern von Relevanz sein können. Dies sind zum einen Informationen über die parteipolitische Zusammensetzung der jeweils amtierenden Regierung. Zum anderen muss eine Variable gebildet werden, welche eine gegebenenfalls bestehende Kongruenz der Koalition mit der parteipolitischen Zusammensetzung der Regierung bzw. der Opposition auf Bundesebene - jeweils zum Zeitpunkt des Regierungsbildungsprozesses auf Landesebene - anzeigt. Für die Bildung der dritten Kontext-Variablen werden Informa- 
tionen über die vor der Wahl seitens der Parteien geäußerten Koalitionspräferenzen benötigt. Eine mögliche Koalition stellt dann eine „Vorwahlallianz“ dar, wenn alle an dieser Koalition beteiligten Parteien vor der Wahl ihre Präferenz zur Bildung eben dieser Koalition artikuliert haben. Zum anderen müssen die Parteikombinationen identifiziert werden, die zumindest von einer der jeweils beteiligten Parteien von vornherein als spätere Koalition ausgeschlossen werden. Die Informationen für die beiden letztgenannten Variablen werden aus den Wahlkampfanalysen der Landtagswahlen entnommen, die in der „Zeitschrift für Parlamentsfragen“ veröffentlicht werden.

Im dritten Schritt müssen die Positionen und politikfeldspezifischen Salienzen der deutschen Parteien auf Bundes- und Landesebene seit der Bundestagswahl 1990 ermittelt werden. Dazu wird auf das von Laver, Benoit und Garry (2003) entwickelte Wordscore-Verfahren zurückgegriffen. Das Verfahren geht von der Beobachtung aus, dass die Wortwahl politischer Akteure in ihren programmatischen Dokumenten oder Reden nicht nach dem Zufallsprinzip erfolgt. Vielmehr senden beispielsweise Parteien in ihren Wahlprogrammen ,ideologische Signale“ (Pappi/Shikano 2004), um ihre Anhängerschaft vor der Wahl zu mobilisieren. Daher nehmen Laver, Benoit und Garry (2003) an, dass man allein aufgrund der relativen Worthäufigkeit eines Dokuments auf dessen programmatische Position oder auch die jeweilige Bedeutung eines Politikfeldes schließen kann. Das Grundprinzip von wordscore ist daher, die relative Worthäufigkeit eines Textes, dessen Position bzw. Salienz man kennt (sogenannte „Referenztexte“), mit der relativen Häufigkeit jedes Wortes eines unbekannten Textes zu vergleichen. Da die Position bzw. Salienz des Referenztexts bekannt ist, kann auf die Position bzw. Salienz des neuen Textes geschlossen werden. Als Referenzwerte können beispielsweise Ergebnisse von Expertenbefragungen dienen (vgl. Benoit/Laver 2006).

Eine große Bedeutung kommt der Auswahl der Referenztexte zu. Diese sollten erstens über eine ähnliche Wortstruktur wie die unbekannten Texte verfügen, zweitens sich inhaltlich auf die Politikfelder beziehen, die Gegenstand der Analyse sind, und drittens möglichst umfangreich sein, um den Anteil informationstragender Wörter zu erhöhen (vgl. Laver et al. 2003: 314-315). Diese Kriterien werden in der Regel von den Wahlprogrammen der Parteien erfüllt. Um die Positionen der deutschen Parteien auf der Landesebene zwischen 1990 und 2007 zu bestimmen, werden als Referenztexte die Wahlprogramme von CDU/CSU, SPD, FDP, Bündnis 90/Die Grünen und der PDS zu zwei Zeitpunkten - zu den Bundestagswahlen 1990 und 2002 - verwendet. Die Referenzpositionen zu den beiden hier berücksichtigten Politikdimensionen - der Wirtschafts- und Sozialpolitik sowie der Gesellschaftspolitik - werden den Expertenbefragungen von Laver und Hunt (1992) sowie Benoit und Laver (2006) entnommen. Die Positionen aus der 1989 durchgeführten Befragung von Laver und Hunt werden den Wahlprogrammen der Parteien zur Bundestagswahl 1990 zugewiesen, die Positionen der 2002er Befragung von Benoit und Laver den Bundestagswahlprogrammen von 2002. Mit der Berücksichtigung von Referenztexten zu Beginn und gegen Ende der Beobachtungsperiode soll sichergestellt werden, dass Themen und politische Schlag- 
wörter, die im Zeitverlauf aufkommen, verschwinden oder aber ihre Bedeutung verändern, gleichermaßen in die Analyse eingehen. ${ }^{6}$

Mithilfe des Wordscore-Verfahrens können die Parteipositionen und die relative Bedeutung der den deutschen Parteienwettbewerb maßgeblich prägenden Konfliktlinien der sozioökonomische Links-Rechts-Konflikt einerseits sowie der Gegensatz zwischen progressiven und konservativen Positionen in gesellschaftspolitischen Fragen andererseits (vgl. Lipset/Rokkan 1967; Pappi 1973) - bestimmt werden. Der Beobachtungszeitraum umfasst alle Wahlen zwischen der Landtagswahl im Saarland vom 28. Januar 1990 und der Bürgerschaftswahl in Bremen vom 13. Mai 2007. Somit liegen der Analyse 69 Landtagswahlen und damit Regierungsbildungsprozesse zugrunde. ${ }^{7}$ Das Ziel ist nun zunächst, ein $\mathrm{Maß}$ zu bestimmen, das die programmatische Distanz zwischen den Parteien adäquat misst. Dazu werden die mit der Wordscore-Methode gewonnenen Positionen und Salienzen der Parteien verwendet.

Gemäß den policy-orientierten Ansätzen der Koalitionsbildung sollte bevorzugt eine Koalitionsregierung gebildet werden, die über eine geringe programmatische Heterogenität verfügt. Wie bestimmt man die Distanz zwischen den Parteien in einem PolicyRaum? Hinich und Munger (1997: 76-83) schlagen die Verwendung euklidischer Distanzen vor. Die euklidische Distanz einer Partei $k$ von einer Partei $l$ ergibt sich aus

$$
d_{k l}=\sqrt{\sum_{j=1}^{m}\left(x_{k j}-x_{l j}\right)^{2}}
$$

wobei $x_{k j}$ für die Position der Partei $k$ auf der Politikdimension $j$ steht. Dabei wird jedoch noch nicht berücksichtigt, dass die Parteien den einzelnen Politikdimensionen jeweils eine unterschiedliche Bedeutung beimessen können. Die gewichtete euklidische

6 Die durch das Wordscore-Verfahren bestimmten Rohwerte der Positionen bzw. Salienzen können nicht unmittelbar mit den entsprechenden Referenzwerten verglichen werden. Laver, Benoit und Garry (2003) schlagen deshalb eine affine Transformation der Rohwerte vor, sodass im Ergebnis Mittelwert und Standardabweichung von geschätzten und Referenzwerten übereinstimmen. Im vorliegenden Fall ist dies unangemessen, da beispielsweise die Streuung der landespolitischen Positionen zwischen 1990 und 2007 nicht notwendigerweise mit der Streuung der bundespolitischen Positionen 1990 und 2005 übereinstimmen muss. Wir verwenden deshalb eine Standardisierung, mit der Mittelwert und Standardabweichung der Bundesparteiprogramme 1990 und 2002 (Referenzwerte) mit denen der (geschätzten) Bundesparteiprogramme 1994 und 1998 übereinstimmen. Für eine detaillierte Beschreibung des Analyseverfahrens und der Daten siehe Bräuninger/Debus (2008).

7 Im Untersuchungszeitraum gibt es eine geringe Anzahl von Regierungsbildungen, die nicht unmittelbar auf Landtagswahlen folgen. Da sämtliche dieser Regierungen nur für kurze Zeit - bis zum regulären oder vorgezogenen Ende der Legislaturperiode - im Amt waren, werden diese Übergangsregierungen im Weiteren nicht berücksichtigt. 
Distanz von Partei $k$ zu Partei $l$ ergibt sich durch das Einbeziehen der relativen Salienzen ${ }^{8}$ :

$$
d_{k l}^{s}=\sqrt{\sum_{j=1}^{m} s_{k j}\left(x_{k j}-x_{l j}\right)^{2}},
$$

wobei der Faktor $s_{k j}$ die relative Bedeutung der Politikdimension $j$ aus der Perspektive von Partei $k$ wiedergibt (vgl. Hinich/Munger 1997: 80). Dies impliziert, dass die von Partei $k$ wahrgenommene Distanz zu anderen Parteien von der Bedeutung, die sie den einzelnen Politikbereichen beimisst, abhängt. Ein Maß für die programmatische Heterogenität einer Koalition ist dann die durchschnittliche gewichtete euklidische Distanz zwischen allen Koalitionspartnern. Die gewichtete ideologische Heterogenität $h$ einer beliebigen Parteienkombination $S$ ergibt sich somit aus:

$$
h(S)=\frac{1}{\# S(\# S-1)} \sum_{\substack{k, l) \in S \times S \\ k \neq l}} d_{k l}^{s},
$$

wobei \#S die Anzahl der Parteien angibt, die der Koalition $S$ angehören. Der resultierende Wert gibt die mit der relativen Salienz gewichtete ideologische Heterogenität einer Parteienkombination wieder.

Das statistische Schätzmodell, das für die Analyse verwendet wird, beruht auf bedingten Wahrscheinlichkeiten (conditional logit; vgl. McFadden 1974; Alvarez/Nagler 1998). Die abhängige Variable umfasst Informationen darüber, welche von allen möglichen Parteikombinationen sich nach einer Landtagswahl in einem Bundesland gebildet hat. Mithilfe dieser Methode wird die Bedeutung der eben beschriebenen unabhängigen Variablen in Form von Regressionskoeffizienten empirisch geschätzt. Alle möglichen Parteikombinationen, die gebildet werden können, werden in der bedingten logistischen Regression mit bestimmten Merkmalen wie der gewichteten ideologischen Heterogenität, den office-orientierten koalitionstheoretischen Kriterien, der möglicherweise bestehenden Vorwahlallianz oder der Übereinstimmung mit den Mustern von Regierung und Opposition auf Bundesebene beschrieben. Die geschätzten Koeffizienten geben dann Aufschluss darüber, wie stark der Einfluss der eingeschlossenen Merkmale unter Kontrolle der übrigen unabhängigen Variablen auf die Koalitionsbildung insgesamt ist.

8 Um die relative Bedeutung einer Politikdimension $j$ für eine Partei $k$ zu bestimmen, wird der mit wordscores bestimmte (absolute) Salienzwert, den Partei $k$ der Dimension $j$ zuweist, normalisiert:

$s_{k j}=s_{k j}^{a b s} / \sum_{v=1}^{m} s_{k v}^{a b s}$.

Nimmt $s_{k j}$ einen Wert von 1 an, so ist der Politikbereich $j$ für Partei $k$ der einzig bedeutsame, liegt er bei 0 , so ist der Bereich vollkommen unbedeutend für die Partei. 


\section{Analyse}

Bevor im Folgenden auf die Ergebnisse der Regressionsanalyse eingegangen wird, werden zunächst die verwendeten Daten näher beschrieben. In einem ersten Schritt beabsichtigen wir anhand von zwei Beispielen zu zeigen, dass sich Landesparteien in der Tat in ihrer programmatischen Ausrichtung unterscheiden und eine differenzierte Betrachtung neue Einsichten in die Koalitionsbildung im deutschen Mehrebenensystem liefern kann. In einem zweiten Schritt wird dann zu untersuchen sein, inwieweit sich mögliche und tatsächlich gebildete Koalitionen hinsichtlich der einzelnen Faktoren unterscheiden.

\subsection{Programmatische Positionierung von Landesparteien: zwei Beispiele}

Es gilt zunächst zu begründen, warum wir anstelle der programmatischen Positionen der Bundesparteien eine Inhaltsanalyse von Wahlprogrammen der Landesparteien vornehmen, um deren spezifische programmatische Haltung zu bestimmen. Wie wir oben bereits argumentiert haben, gehen viele qualitativ-politikwissenschaftliche wie auch historische Studien davon aus, dass sich die ideologischen Ausrichtungen sowohl zwischen Landes- und Bundesparteien als auch zwischen den einzelnen Landesverbänden einer Partei unterscheiden können. Die Ursachen hierfür werden in langfristig prägenden sozialstrukturellen Merkmalen der Wählerschaft der jeweiligen historischen Regionen bzw. der Bundesländer gesehen. Zur Bestimmung der ideologischen Position jeder einzelnen Landespartei zu einer Wahl wurde mit wordscore eine Inhaltsanalyse von 306 Wahlprogrammen durchgeführt. ${ }^{9}$ Die folgenden Abbildungen 2 und 3 geben die programmatischen Positionen der Parteien im Land Baden-Württemberg und im Stadtstaat Bremen als zwei sozialstrukturell wie parteipolitisch sehr unterschiedlich geprägten Bundesländern wieder. Im Gegensatz zum norddeutschen, urbanen und von der SPD dominierten Land Bremen ist der 1952 gegründete Südweststaat Baden-Württemberg eher ländlich geprägt und wird parteipolitisch von den Christdemokraten dominiert (vgl. Scherer 2002; Wehling 2002). Berücksichtigt werden in den beiden Abbildungen nur die vier ,zentralen“ und systemimmanenten Parteien CDU, SPD, FDP und Bündnis 90/ Die Grünen. Es wird erstens deutlich, dass die Parteien in Bremen gesellschaftspolitisch progressivere Positionen einnehmen als die baden-württembergischen Landesparteien. Dies kann als ein Hinweis für den Einfluss sozialstruktureller Gegebenheiten eines

9 Die Beschaffung von Wahlprogrammen gestaltet sich insbesondere im Fall von kleineren und/ oder links- bzw. rechtsextremen Parteien als schwierig. Wir verwenden daher im Fall von Republikanern (REP), Nationaldemokraten (NPD), der Schill-Partei und der Deutschen Volksunion (DVU) die programmatische Position der jeweiligen Bundespartei, die in der Expertenbefragung von Benoit und Laver (2006) angegeben ist, als Näherungsindikator. Für drei landesspezifische Parteien - die „Arbeit für Bremen und Bremerhaven“ (AFB), die Hamburger „STATT Partei“ und den „Südschleswigschen Wählerverband“ (SSW) - liegen die Wahlprogramme hingegen weitgehend vor. Im Fall des SSW werden die ermittelten programmatischen Positionen von 2000 verwendet, um die Position des SSW für die Landtagswahlen 1992 und 1996 zu bestimmen. Auch im Fall der CDU Brandenburg liegt deren Wahlprogramm von 1990 nicht vor; als Schätzung dienen hier die Werte von 1994. 
Abbildung 2: Programmatische Parteienkonstellation in Baden-Württemberg von 1992 bis 2006

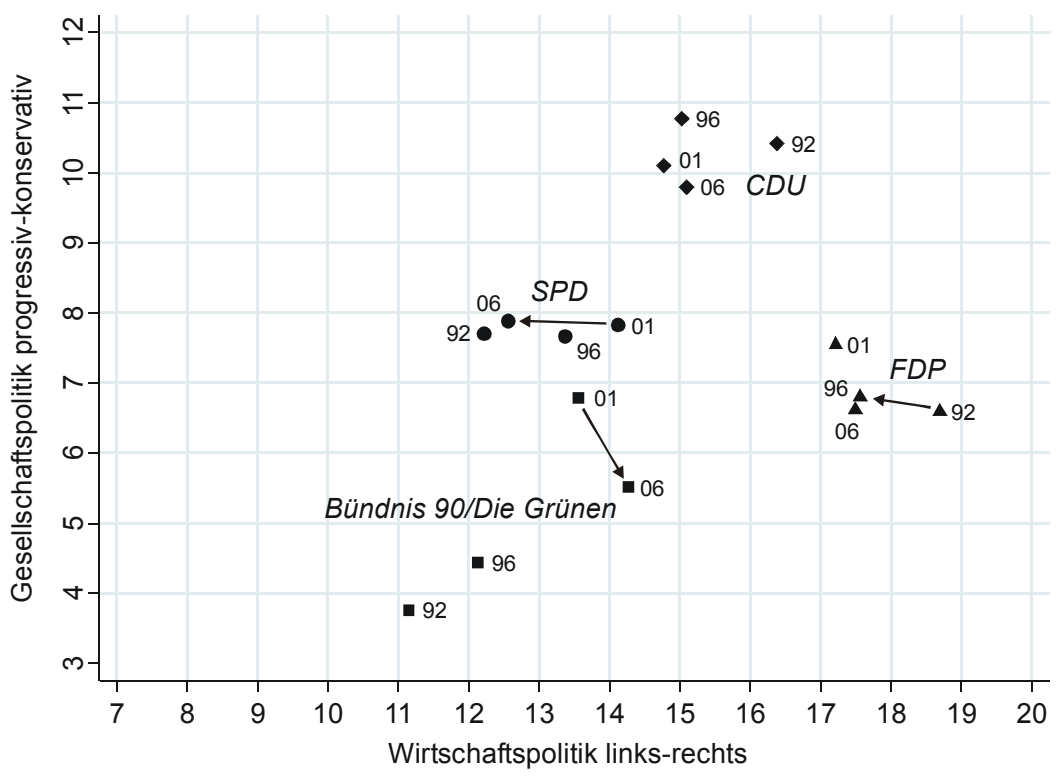

Abbildung 3: Programmatische Parteienkonstellation in Bremen von 1991 bis 2007

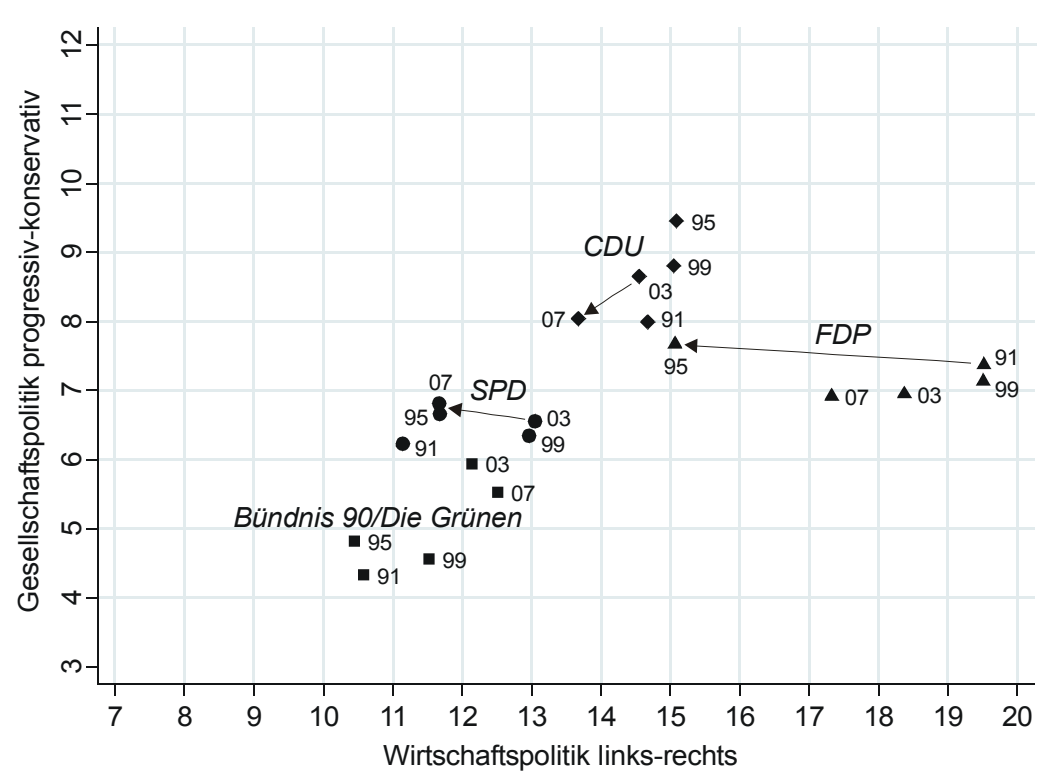


Landes auf die programmatische Ausrichtung von Parteien gewertet werden, da man von Parteien, die um Stimmen von primär städtisch und damit eher gesellschaftspolitisch progressiv geprägten Wählern werben, liberalere Haltungen in Fragen der Gesellschaftspolitik erwarten würde als von Parteien, die im Wettbewerb um die Stimmen von überdurchschnittlich vielen ländlich-konservativ geprägten Wählern stehen.

Es wird zweitens deutlich, dass die Positionen der Parteien im Zeitverlauf nicht gleich bleiben, sondern sich - mehr oder weniger stark - verändern. Solche Veränderungen, die im Hinblick auf den Koalitionsbildungsprozess von Relevanz sein könnten, sind in den beiden Abbildungen mit Richtungspfeilen markiert. Im Fall der baden-württembergischen Parteien fallen drei programmatische Bewegungen besonders ins Auge. Dies ist zum einen die Veränderung in der FDP-Position zwischen 1992 und 1996 hin zu einer wirtschaftspolitisch moderateren Haltung. Dies mag als ein Annäherungsversuch an die CDU gewertet werden, da eine Fortsetzung der von 1992 bis 1996 bestehenden Großen Koalition weder von Sozial- noch von Christdemokraten gewünscht wurde und so die FDP die Chancen auf eine Regierungsbildung mit der CDU nach 30 Jahren in der Opposition durch eine programmatische Annäherung an die Union erhöhte (vgl. Sturm 1996: 272-273). Die zwei weiteren Auffälligkeiten betreffen die Positionierung von SPD und Grünen zur Landtagswahl 2006. Nachdem die Sozialdemokraten sich leicht, aber stetig wirtschaftspolitisch nach rechts bewegt haben, nahmen sie zur Wahl 2006 eine Position ein, die der von 1992 entsprach. Dies mag als eine Reaktion auf die Konkurrenz von links durch das erstmalige Antreten der „Wahlalternative Arbeit \& soziale Gerechtigkeit e. V.“ (WASG) mit dem früheren SPD-Landesvorsitzenden Ulrich Maurer als deren Spitzenkandidaten gewertet werden. Koalitionspolitisch auffällig ist der Weg zu wirtschaftspolitisch moderateren Positionen bei den baden-württembergischen Grünen. Gab es bereits nach den Landtagswahlen 1992 schon - letztlich erfolglose - Sondierungsgespräche zwischen den dort als „strukturkonservativ“ geltenden Grünen und dem CDU-Landesverband, so gilt laut Schneider (2001: 392) die Bildung einer schwarz-grünen Koalition in Baden-Württemberg als nicht unwahrscheinlich. Dies kann seitens der Südwest-Grünen als Anreiz verstanden werden, sich der Union programmatisch zu nähern, um die Bildung einer solchen Koalition zu erleichtern.

In Bremen treten ebenfalls drei Bewegungen der dortigen Landesparteien besonders hervor. Dies ist zum einen die Positionsverschiebung der FDP nach links zu den Wahlen 1995. Zu diesem Zeitpunkt befanden sich die Liberalen in einer Koalitionsregierung mit SPD und Grünen. Für die Zeit nach der Wahl favorisierte die FDP eine Koalition mit der CDU und der neu gegründeten „Arbeitsgemeinschaft für Bremen und Bremerhaven“ (AFB). Die programmatische Annäherung an die CDU kann als Vorbereitung eines solchen Bündnisses angesehen werden. Zum anderen ist die programmatische Haltung der CDU zu den Bürgerschaftswahlen 2007 auffällig. Das Wahlprogramm der Bremer Christdemokraten 2007 ist im Untersuchungszeitraum von 1991 bis 2007 das wirtschaftspolitisch am weitesten links angesiedelte und gesellschaftspolitisch progressivste dieser Landespartei. Was können die Ursachen für eine solche atypische Positionierung einer Unionspartei sein? Ein Grund mag in dem Wunsch gelegen haben, die seit 1995 bestehende Koalition mit der SPD fortzusetzen und dies mit einer Annäherung an die Position der Sozialdemokraten zu begünstigen. Ähnlich wie in Baden-Württemberg bewegten sich auch die Bremer Sozialdemokraten bei der Wahl 2007 wirtschaftspoli- 
tisch nach links, was auch hier am erstmaligen und letztlich erfolgreichen Antreten der PDS gelegen haben mag.

Ausgehend von diesen Befunden kann man durchaus von verschiedenen Formen des ideologischen Parteienwettbewerbs in den Bundesländern sprechen, je nachdem, ob sozialstrukturelle Veränderungen das Auftreten neuer Parteien oder die Aussicht auf Regierungsbeteiligung die inhaltliche Ausrichtung der Parteien dominiert. Eine solche Interpretation macht jedoch die bereits erwähnte Endogenitätsproblematik deutlich. Verändern Parteien ihre programmatischen Positionen bereits mit Hinblick auf das (gewünschte) Ergebnis des Regierungsbildungsprozesses, dann stellt streng genommen die ideologische Heterogenität einer Parteienkonstellation keinen unabhängigen Erklärungsfaktor der Regierungsbildung dar. Andererseits spiegeln die von den Parteitagsdelegierten verabschiedeten Wahlprogramme die offiziellen Politikziele der Landesparteien für die folgende Legislaturperiode wider. Es erscheint daher sinnvoll, die aus diesen Dokumenten generierten programmatischen Positionen der Landesparteien für die Analyse der Bestimmungsfaktoren der Regierungsbildung in den Bundesländern zu verwenden. Um zu testen, ob die programmatischen Standpunkte der Landesparteien eine höhere Erklärungskraft aufweisen als die aus Bundestagswahlprogrammen generierten und den Landesparteien zugewiesenen Positionen, werden die Regressionsmodelle mit zwei unterschiedlichen Heterogenitätsmaßen geschätzt, wobei das eine auf den programmatischen Positionen der jeweiligen Landes- und das andere auf den Politikzielen der Bundesparteien beruht. Bevor die Ergebnisse der Analyse dargestellt werden, wird im folgenden Abschnitt detaillierter auf die Operationalisierung und Beschreibung der weiteren unabhängigen sowie der abhängigen Variablen näher eingegangen.

\subsection{Parteipolitische Zusammensetzung von Regierungskoalitionen in den Ländern}

Im Beobachtungszeitraum von 1990 bis 2007 fanden in den Ländern insgesamt 69 Wahlen zu den deutschen Landesparlamenten statt. Für die meisten Bundesländer liegen aufgrund einer fünfjährigen Wahlperiode damit vier Wahlen vor. Für Bremen, Mecklenburg-Vorpommern und Sachsen-Anhalt fallen in den Beobachtungszeitraum aufgrund einer vierjährigen Wahlperiode fünf Wahlen, ebenso wie in Hamburg, wo die für ungültig erklärte Wahl zur Hamburger Bürgerschaft von 1991 zu Neuwahlen im November 1993 führte, und in Berlin, wo nach dem Bruch der Großen Koalition unter Eberhard Diepgen (CDU) die PDS-tolerierte rot-grüne (erklärte) Übergangsregierung von Klaus Wowereit vorgezogene Neuwahlen im Oktober 2001 herbeiführte.

Was den Beginn und das Ende einer Regierung ausmacht, ist in der Literatur nicht unumstritten (vgl. King et al. 1990; Diermeier/Stevenson 1999), da die schlichte Fortführung einer Koalition nach einer Wahl auch als ,pfadabhängiges“ und nicht als unabhängiges Ereignis verstanden werden kann. Allerdings hängt die Beantwortung dieser Frage auch von der Art der zugrunde liegenden Forschungsfrage ab. So ist es bei einer Untersuchung der Karriereverläufe politischer Eliten durchaus zweckmäßig, die Amtsdauer eines Mandatsträgers über mehrere Wahlperioden hinweg in Augenschein zu nehmen, also aufzusummieren. Bei einer Untersuchung von Koalitionsbildungsprozessen 
erscheint es hingegen sinnvoll, jede Neuwahl als einen eigenen, aussagekräftigen bzw. erklärungsbedürftigen Fall anzusehen. Dies erscheint vor dem Hintergrund gerechtfertigt, dass Wahlen die Parteien regelmäßig zu einer Neupositionierung in ihrer programmatischen Ausrichtung veranlassen, aber auch neue Mehrheitsverhältnisse nach sich ziehen und damit veränderte Rahmenbedingungen für die Regierungsbildung definieren. Ausgangspunkt für die folgenden Analysen sind deshalb alle 69 Regierungsbildungssituationen, die sich aufgrund von Neuwahlen in den Bundesländern im Zeitraum von 1990 bis 2007 ergaben. Den möglichen positiven Effekt, der von einer zum Zeitpunkt der Regierungsbildung noch im Amt befindlichen Regierung ausgeht, kontrollieren wir durch eine Variable, die wir als einen kontextuellen Faktor in die statistische Analyse einschließen. Innerhalb einer Wahlperiode wurden im Untersuchungszeitraum keine Veränderungen der parteipolitischen Zusammensetzungen von Landesregierungen vorgenommen, lässt man einmal die von Beginn an zeitlich begrenzt geschlossenen Koalitionen, wie die Berliner rot-grüne Koalition von 2001 oder die sozial-liberale Koalition in Bremen unter Klaus Wedemeier, die von Februar bis Juli 1995 amtierte, außer Acht. Konsequenterweise sehen wir ebenfalls davon ab, das bloße Auswechseln von Ministerpräsidenten oder gar Ministern als einen weiteren Fall der Regierungsbildung zu definieren.

Unser Augenmerk bei der Analyse der Regierungsbildung liegt auf der parteipolitischen Zusammensetzung der Landesregierungen. Als Regierungspartner kommen dafür alle Parteien in Betracht, die in einem Landesparlament vertreten sind, als Regierungen entsprechend alle Kombinationen dieser Parteien. Dabei erscheint es zunächst unerheblich, ob eine entsprechende Regierung die Unterstützung einer Mehrheit von Abgeordneten besitzt oder als sogenannte Minderheitsregierung ins Amt kommt. Im internationalen Vergleich sind Minderheitsregierungen so häufig, dass kaum noch von einer Abweichung von der Regel gesprochen werden kann (vgl. Strøm 1990b; Laver/ Schofield 1998: 70-74). Die Bildung der deutschen Bundesregierungen ist diesbezüglich eher eine Ausnahme. Auch in den deutschen Ländern sind Minderheitsregierungen selten. Im Untersuchungszeitraum bestanden mit den PDS-tolerierten Regierungen in Sachsen-Anhalt unter Ministerpräsident Höppner (SPD) lediglich zwei auf längere Dauer angelegte Minderheitsregierungen. Bei drei im Landtag vertretenen Parteien gibt es somit $2^{3}-1=7$ mögliche Parteikombinationen, einschließlich der Einparteiregierungen und der Allparteienkoalition. Die höchste Parteienanzahl weisen der im September 2004 gewählte vierte sächsische Landtag und die sechzehnte Bremer Bürgerschaft, die im Mai 2007 gewählt wurde, mit jeweils sechs vertretenen Parteien auf, was die Menge der theoretisch möglichen Parteiregierungen auf $2^{6}-1=63$ erhöht. Insgesamt ergibt sich für die 69 Ereignisse der Regierungsbildung eine Menge von 1215 Koalitionsoptionen. $\mathrm{Ta}$ belle 1 gibt einen Überblick über alle möglichen und die tatsächlich gebildeten Koalitionen in den Bundesländern im Zeitraum von 1990 bis 2007.

Ein Vergleich von möglichen und tatsächlich gebildeten Koalitionen erlaubt erste Rückschlüsse über die Determinanten der Regierungsbildung in den Bundesländern. Zunächst fällt auf, dass bei der Vielzahl möglicher Koalitionen dennoch in 30 von 65 Fällen und damit in nahezu der Hälfte der Fälle die Vorgängerregierung fortgesetzt 


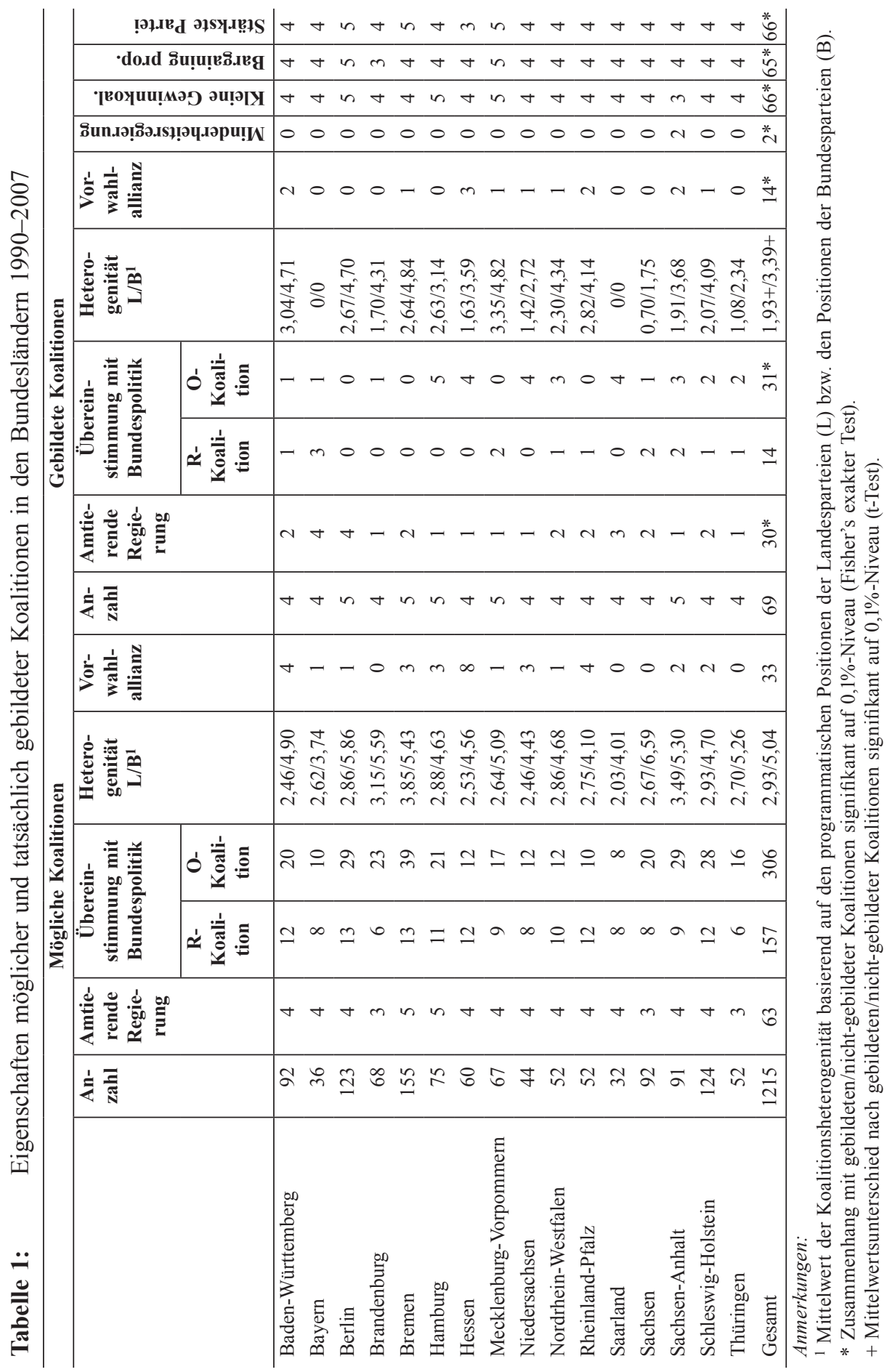


wird. ${ }^{10}$ Dies trifft für Bayern im gesamten Zeitraum zu, aber auch in Berlin und im Saarland waren Koalitionswechsel eher selten, während in den ostdeutschen Ländern Regierungen ihre Arbeit regelmäßig nicht über die Wahlperiode hinaus fortsetzen konnten. Weiterhin kongruieren 45 der 69 gebildeten Regierungen mit den Mehrheitsverhältnissen auf Bundesebene. Differenziert man nach der parteipolitischen Übereinstimmung der gebildeten Landesregierungen mit den Mustern von Regierung und Opposition auf Bundesebene, so fügen sich 14 Landesregierungen in das Lager der R-Länder und 31 in die Gruppe der O-Länder im Bundesrat ein. Dies trifft natürlich immer dann zu, wenn eine Einparteiregierung vorliegt, so insbesondere in Bayern und im Saarland; eine gehäufte Kongruenz von Landes- und Bundesregierung ergibt sich auch für Niedersachen und Schleswig-Holstein. Stets dem bundespolitischen Trend entgegengesetzt wurden hingegen Koalitionen in Berlin und Bremen gebildet. Insgesamt gab es im Zeitraum 24 Landesregierungen, die dem gemischten Lager im Bundesrat zuzurechnen waren.

Tabelle 1 legt ebenfalls nahe, dass die Heterogenität der Politikpositionen möglicher Koalitionspartner die Chancen für die Bildung einer Koalition verringern. Im Durchschnitt sind in nahezu allen Ländern die Konfliktpotenziale der gebildeten Regierungen weitaus geringer als die der möglichen Koalitionen. Dies gilt trotz des Umstandes, dass die Menge der möglichen Koalitionen auch (konfliktlose) Einpartei- oder (konfliktarme) kleine Bündnisregierungen enthält, die mit dem Verfehlen der Mehrheitshürde kaum gebildet werden dürften. Das gleiche Bild ergibt sich erwartungsgemäß, wenn man zur Berechnung der gewichteten ideologischen Heterogenität nicht die Werte der Landes-, sondern die der Bundesparteien auf der Grundlage von deren Wahlprogrammen zur vorangegangenen Bundestagswahl heranzieht.

Von den Parteien im Vorfeld von Wahlen geäußerte Koalitionspräferenzen bzw. ausgeschlossene Koalitionsoptionen können als weiterer Kontextfaktor untersucht werden. Auch hier zeigt sich in der deskriptiven Gesamtschau der Regierungsbildungen, dass Vorwahlallianzen eine mögliche Bedeutung zukommt (mit 14 realisierten von 33 angekündigten Allianzen), ebenso wie ausgeschlossenen Optionen (mit null realisierten von 546 ablehnenden Äußerungen). ${ }^{11}$ Ähnliches gilt für die einzelnen Office-Faktoren: Die schließlich zustande gekommenen Regierungen bzw. Regierungskoalitionen sind mit zwei Ausnahmen niemals Minderheitsregierungen, zumeist aber kleine Gewinnkoalitionen, enthalten die stimmenstärkste Partei und entsprechen der „bargaining proposition“. Alle diese bivariaten Zusammenhänge zeigen in die erwartete Richtung und sind - abgesehen von der Gruppe der R-Landesregierungen - statistisch signifikant. Ob und welche der einzelnen Faktoren auch unter Kontrolle von jeweils anderen Faktoren relevant für die Koalitionsbildung sind, kann auf der Ebene bivariater Zusammenhänge nicht beurteilt werden. Hierzu bedarf es eines multivariaten Analyseansatzes. Dieser Analyse wenden wir uns im folgenden Abschnitt zu.

10 Für die vier ostdeutschen Länder Brandenburg, Mecklenburg-Vorpommern, Sachsen, SachsenAnhalt und Thüringen kann für die Regierungsbildungsprozesse 1990 naturgemäß keine Vorgängerregierung identifiziert werden. Im Fall von Berlin werten wir die Koalition aus SPD und Alternativer Liste, die von 1989 bis 1990 im Westteil der Stadt bestand, als amtierende Regierungskoalition.

$11 \mathrm{Da}$ es keinen Fall gibt, bei dem eine von vornherein abgelehnte Koalitionsoption dennoch in die Regierungsverantwortung kam, kann diese Variable in den später aufgeführten Regressionsmodellen nicht berücksichtigt werden. 


\subsection{Multivariate Analyse der Determinanten der Koalitionsbildung}

In den Tabellen 2 und 3 sind die Ergebnisse der bedingten logistischen Regressionsanalysen dargestellt, mit denen wir den Einfluss der oben eingeführten Office-, Policy- und Kontextfaktoren auf die Koalitionsbildung in den Ländern bestimmen. ${ }^{12}$ In dieser Form der quantitativen Analyse stellt jede Situation der Regierungsbildung einen einzelnen Untersuchungsfall dar. Die geschätzten Koeffizienten ergeben sich aus dem Vergleich von tatsächlich gebildeten und allen anderen, möglichen Koalitionen (oder Einparteiregierungen) und zeigen an, ob bestimmte Koalitionseigenschaften wie der Einschluss der größten Partei oder die Übereinstimmung mit dem R- oder O-Länder-Regierungstyp die Wahrscheinlichkeit für die Koalition erhöht (mit positivem Koeffizienten) oder erniedrigt (mit negativem Koeffizienten). Während die Bedeutung der Richtung der ausgewiesenen Koeffizienten eindeutig ist, erschließt sich die Bedeutung ihrer absoluten Größe nicht unmittelbar, da bei einer (bedingten) logistischen Regression Koeffizienten und Wahrscheinlichkeiten in einem exponentiellen, also nicht-linearen Zusammenhang stehen. Wir werden deshalb abschließend und beispielhaft diskutieren, welcher Effekt vom bundespolitischen Kontext der Regierungsbildung ausgeht, in welchen Fällen also der bundespolitische Druck, eine kongruente Regierung auf Landesebene zu bilden, das Koalitionsspiel entscheidend veränderte.

Tabelle 2 enthält in vier Modellen die Hauptergebnisse der Studie. Im ersten Modell werden Anreize für die Koalitionsbildung berücksichtigt, die sich aus einer Office- bzw. einer Policy-Orientierung der Landesparteien ergeben. Weiterhin berücksichtigen wir die zentrale Variable der Übereinstimmung mit der bundespolitischen Mehrheitskonstellation sowie - als weiteren Kontextfaktor - die Kongruenz mit der amtierenden Regierung des Landes. Dabei ist zu beachten, dass die letztgenannte Variable faktisch die um eine Wahlperiode zeitverzögerte abhängige Variable darstellt und damit die Bedeutung von allen anderen relevanten Faktoren unter Umständen geringer aussehen lässt, als sie tatsächlich ist. Schließlich ist auch die Vorgängerregierung aufgrund bestimmter Faktoren zustande gekommen. Wenn nun die Nachfolgeregierung bei unveränderten Randbedingungen wieder in der gleichen parteipolitischen Zusammensetzung zusammentritt, dann spiegelt sich dies teilweise in einem größeren, positiven Koeffizienten für die „amtierende Regierung“ wider, während die Effekte aller anderen Faktoren unterschätzt werden können. ${ }^{13}$ Dennoch beziehen wir die Vorgängerregierung mit ein, da mit der Vertrautheit der Koalitionspartner und geringeren Transaktionskosten der Regierungsbildung auch dem Status quo eine inhaltliche Bedeutung zukommt. Das zweite Modell enthält mit der Vorwahlallianz einen weiteren Kontextfaktor der Regierungsbildung, und mit dem dritten Modell soll der teilweisen Endogenität der Vorwahlallianz Rechnung getragen werden. Das vierte Modell schließlich repliziert Modell 3, wobei die

12 Die verwendeten Wahlprogramme der Landes- und Bundesparteien sind im frei zugänglichen Online-Archiv Polidoc.Net (http://polidoc.net) hinterlegt; der Replikationsdatensatz für die nachfolgenden Analysen kann von den Autoren bezogen werden (http://www.tbraeuninger.de/ download.html).

13 Zum Problem der Einbeziehung von zeitverzögerten Variablen (lagged dependent variables) bei Autokorrelation der Residuen vgl. Achen (2000) sowie Keele/Kelly (2006). 
Tabelle 2: Bedingte logistische Regression der Koalitionsbildung in den deutschen Bundesländern von 1990 bis 2007

\begin{tabular}{|c|c|c|c|c|}
\hline \multirow[t]{2}{*}{ Attribute } & \multicolumn{4}{|c|}{ Modelle } \\
\hline & (1) & (2) & (3) & (4) \\
\hline \multicolumn{5}{|l|}{ Office-Faktoren } \\
\hline Minderheitsregierung & $\begin{array}{l}-1,01 \\
(1,23)\end{array}$ & $\begin{array}{l}-0,30 \\
(1,42)\end{array}$ & $\begin{array}{l}-1,33 \\
(1,31)\end{array}$ & $\begin{array}{l}-1,75 \\
(1,40)\end{array}$ \\
\hline Kleine Gewinnkoalition & $\begin{array}{l}3,21 \\
(1,39)^{* *}\end{array}$ & $\begin{array}{l}3,97 \\
(1,42)^{* * *}\end{array}$ & $\begin{array}{l}3,71 \\
(1,46)^{* *}\end{array}$ & $\begin{array}{l}3,17 \\
(1,41)^{* *}\end{array}$ \\
\hline Bargaining proposition & $\begin{array}{c}0,92 \\
(1,02)\end{array}$ & $\begin{array}{c}0,59 \\
(0,86)\end{array}$ & $\begin{array}{c}0,91 \\
(0,92)\end{array}$ & $\begin{array}{c}1,05 \\
(1,04)\end{array}$ \\
\hline Stärkste Partei & $\begin{array}{l}1,53 \\
(0,61)^{* *}\end{array}$ & $\begin{array}{l}1,58 \\
(0,64)^{* *}\end{array}$ & $\begin{array}{l}1,43 \\
(0,60)^{* *}\end{array}$ & $\begin{array}{c}1,14 \\
(0,65)^{*}\end{array}$ \\
\hline \multicolumn{5}{|l|}{ Policy-Faktoren } \\
\hline Heterogenität Landesparteien & $\begin{array}{l}-0,25 \\
(0,10)^{* *}\end{array}$ & $\begin{array}{l}-0,28 \\
(0,12)^{* *}\end{array}$ & $\begin{array}{l}-0,28 \\
(0,12)^{* *}\end{array}$ & \\
\hline Heterogenität Bundesparteien & & & & $\begin{array}{l}-0,31 \\
(0,08)^{* * *}\end{array}$ \\
\hline \multicolumn{5}{|l|}{ Kontextuelle Faktoren } \\
\hline Amtierende Regierung & $\begin{array}{l}1,92 \\
(0,52)^{* * *}\end{array}$ & $\begin{array}{c}1,27 \\
(0,63)^{* *}\end{array}$ & $\begin{array}{l}2,28 \\
(0,53)^{* * *}\end{array}$ & $\begin{array}{l}2,00 \\
(0,53)^{* * *}\end{array}$ \\
\hline \multicolumn{5}{|l|}{ Übereinstimmung mit Bundespolitik } \\
\hline R-Koalition & $\begin{array}{c}0,78 \\
(0,64)\end{array}$ & $\begin{array}{c}0,48 \\
(0,64)\end{array}$ & $\begin{array}{l}1,20 \\
(0,57)^{* *}\end{array}$ & $\begin{array}{c}0,74 \\
(0,56)\end{array}$ \\
\hline O-Koalition & $\begin{array}{l}1,39 \\
(0,47)^{* * *}\end{array}$ & $\begin{array}{c}0,92 \\
(0,52)^{*}\end{array}$ & $\begin{array}{c}1,32 \\
(0,63)^{* *}\end{array}$ & $\begin{array}{c}0,80 \\
(0,60)\end{array}$ \\
\hline Vorwahlallianz & & $\begin{array}{l}2,29 \\
(1,08)^{* *}\end{array}$ & & \\
\hline Vorwahlallianz-Residuum & & & $\begin{array}{c}1,23 \\
(0,53)^{* *}\end{array}$ & $\begin{array}{l}0,84 \\
(0,33)^{* * *}\end{array}$ \\
\hline $\mathrm{N}$ & 1215,00 & 1215,00 & 1215,00 & 1215,00 \\
\hline Pseudo $\mathrm{R}^{2}$ & 0,72 & 0,74 & 0,75 & 0,75 \\
\hline Log pseudolikelihood & $-52,26$ & $-48,13$ & $-46,19$ & $-46,37$ \\
\hline AIC & 120,52 & 114,26 & 110,38 & 110,74 \\
\hline $\mathrm{BIC}$ & 161,34 & 160,19 & 156,30 & 156,66 \\
\hline IIA-Test & 0,892 & 0,976 & 0,824 & 0,944 \\
\hline $\begin{array}{l}\text { Anteil korrekt vorhergesagter } \\
\text { Koalitionen }\end{array}$ & $75,4 \%$ & $75,4 \%$ & $78,3 \%$ & $75,4 \%$ \\
\hline
\end{tabular}

sachpolitische Heterogenität der Koalitionen auf der Basis von Bundestags- anstelle von Landtagswahlprogrammen bestimmt wird.

Die einzelnen Modelle vermögen die Koalitionsbildung in den Bundesländern erfreulich gut abzubilden. Alle Modelle sind hoch signifikant und der Vergleich der Werte des Akaike-Informationskriteriums (AIC) ergibt, dass die Spezifizierung der jeweils komplexeren Modelle 2 und 3 sinnvoll und gerechtfertigt ist. Der von Hausman und McFadden (1984) vorgeschlagene IIA-Test ergibt für alle vier Modelle, dass von einer 
Unabhängigkeit der Koalitionsalternativen ausgegangen werden kann. ${ }^{14}$ Die Erklärungskraft der Modelle ist durchweg hoch. Bereits das erste Modell vermag 75,4 Prozent der tatsächlich gebildeten Koalitionen richtig vorauszusagen, für die Modelle 2 und 3 ergibt sich eine korrekte Vorhersage in 75,4 Prozent bzw. 78,3 Prozent der Fälle. Als erstes Ergebnis der Untersuchung kann deshalb festgehalten werden, dass das bisweilen in der Literatur geäußerte Unbehagen über den vermeintlich geringen Erkenntnisgewinn durch formale Theorien der Koalitionsbildung (vgl. Kropp et al. 2002: 10) zumindest für den Fall der deutschen Länder durch eine differenzierte Bewertung ersetzt werden sollte. Pappi, Becker und Herzog (2005: 453) konnten anhand ihres Modells, das zwar alle Regierungsbildungsprozesse auf Landesebene von der Gründung der Bundesrepublik bis 2003 umfasste, jedoch keine landesspezifische Messung der Parteipositionen beinhaltete, ebenfalls mit 61 Prozent einen hohen Anteil an Koalitionen korrekt voraussagen. Dennoch ist sicherlich richtig, dass einzelne Theorien in einer isolierten Betrachtung nur wenig Erklärungskraft haben. Dies ist jedoch kaum verwunderlich, da reale Regierungsbildungsprozesse - wie andere soziale Prozesse auch - von einer Vielzahl von individuellen und systemischen Faktoren beeinflusst werden. Bemerkenswert ist mithin, dass die Zusammenschau von bereits wenigen, durchweg einfachen und allgemeinen Theorien der Koalitionsbildung über drei Viertel der Regierungsbildungen abzubilden vermag.

Die Ergebnisse der Regressionsanalysen geben ein recht klares Bild über die Determinanten der Regierungsbildung auf Länderebene. Erstens scheint das Streben der Parteien nach Regierungsämtern, auch unabhängig von der parteipolitisch-ideologischen Ausrichtung der Koalition, eine Rolle zu spielen. Kleine Gewinnkoalitionen haben deshalb in allen Modellen signifikant höhere Chancen zustande zu kommen. Das Gleiche gilt für Koalitionen, welche die stärkste Partei einschließen. Wie in Tabelle 1 bereits zu sehen war, sind realiter 66 von 69 Regierungskoalitionen kleine Gewinnkoalitionen bzw. umfassen die stimmenstärkste Partei im Landesparlament. Beide Faktoren können deshalb geradezu als notwendige Bedingungen charakterisiert werden; angesichts der Vielzahl von solchen Koalitionen sind diese aber mitnichten hinreichend in ihrer Erklärungskraft. An dieser Stelle kommt unter anderem der ideologischen Heterogenität der Koalition eine Bedeutung zu. Zweitens führt nämlich ein erhöhter Policy-Konflikt zwischen den potenziellen Koalitionspartnern zu einer Verringerung der Koalitionswahrscheinlichkeit. Dies steht in Übereinstimmung mit Axelrods (1970) Argument, dass Akteure, deren inhaltliche Politikvorstellungen stark entgegengerichtet sind, sich seltener auf eine gemeinsame Regierungspolitik werden einigen können (und wenn sie dies tun, öfter und schneller auseinanderbrechen).

Drittens haben die Kontextfaktoren einen Einfluss auf die Koalitionsbildung. Im ersten Modell wird mit der ,amtierenden Regierung“ der Status quo auf Landesebene und mit der „Übereinstimmung mit der Bundespolitik“ der Status quo auf der Bundesebene berücksichtigt. Beide Faktoren haben den erwarteten positiven Effekt. In Modell 2 wer-

14 Diese Annahme wäre verletzt, wenn die relative Wahrscheinlichkeit, eine Koalition A gegenüber einer Koalition B vorzuziehen, von der Anwesenheit einer dritten Koalition C abhängig ist. Eine Folge davon wären inkonsistente Parameterschätzungen (Hausman/McFadden 1984; vgl. Martin/Stevenson 2001: 39). 
den weiterhin die von den Parteien im Vorfeld der Landtagswahlen geäußerten Koalitionspräferenzen einbezogen. Auch die Variable „Vorwahlallianz“ hat den erwarteten positiven und signifikanten Effekt auf die Koalitionswahrscheinlichkeit. Dabei fällt auf, dass mit der Berücksichtigung der Vorwahlallianz die Bedeutung des landes- sowie des bundespolitischen Status-quo-Faktors sinkt. Dies ist allerdings wenig verwunderlich, da die Koalitionspräferenz vor der Wahl ja bereits vor dem damaligen landes- und bundespolitischen Hintergrund formuliert wurde. Auch stellt Modell 2 gegenüber Modell 1 nur bedingt eine Verbesserung dar. Modell 2 ist zwar nach dem Likelihood-Ratio-Test, dem AIC- und dem BIC-Kriterium gegenüber Modell 1 vorzuziehen; letztlich sagen jedoch beide Modelle einen gleich hohen Anteil an Koalitionen korrekt vorher.

Im dritten Modell soll deshalb versucht werden, der vermuteten kausalen Verflechtung der Faktoren Rechnung zu tragen. Vorwahlallianz und Koalitionswahl lassen sich dabei schon aus Gründen der zeitlichen Abfolge in eine eindeutige Kausalkette stellen, sodass eine Form der Pfadanalyse zur Anwendung kommt. ${ }^{15}$ Dazu wird in einem ersten Schritt eine logistische Regressionsanalyse der Vorwahlallianz durchgeführt, wobei alle anderen Regressoren von Modell 1 als unabhängige Variablen dienen. Die Ergebnisse unterstützen die Vermutung, dass die Vorwahlallianz selbst von den Faktoren der Regierungsbildung abhängt. Signifikante Effekte finden sich für die amtierende Regierung und die Übereinstimmung mit der Bundespolitik. In einem zweiten Schritt wird dann in Modell 2 die Variable der Vorwahlallianz durch die Devianz-Residuen des Logit-Modells ersetzt. ${ }^{16}$ Im Ergebnis erhält man damit eine Abschätzung des „Netto-Effekts“ der Vorwahlallianz, der nicht von den anderen Determinanten des Koalitionsspiels herrührt, sowie eine bessere Abschätzung der Effektgröße dieser anderen Faktoren (Modell 3). Es bestätigt sich zum einen, dass die beiden Kontextfaktoren einen wichtigen Einfluss haben (mit Effektkoeffizienten von 2,28 für die amtierende Regierung und 1,20 bzw.

15 Während für die Analyse von Pfadmodellen mit ausschließlich kategorialen Variablen auf loglineare Pfadmodelle (siehe z.B. Klein 2005) und für Modelle mit kontinuierlichen endogenen Variablen auf zweistufige Schätzverfahren (two-stage least square) zurückgegriffen werden kann (siehe z.B. Hallermann/Edinger 2001), liegen keine entsprechende Verfahren zur Bestimmung von direkten und indirekten Effekten für Modelle mit dichotomen Variablen vor (für ein ähnliches, sogenanntes Two-stage-probit-least-squares-Verfahren [2SPLS] vgl. Achen 1986; Alvarez/Glasgow 1999). Zu beachten ist, dass 2SPLS zwar eine konsistente Schätzung der Koeffizienten erlaubt, unter Umständen aber verzerrte Standardfehler berichtet. Da eine direkte Korrektur der Standardfehler nicht möglich ist (Achen 1986: 42), sollten die hier berichteten Fehler mit Vorsicht interpretiert werden.

16 Für die logistische Regression ergeben sich folgende Koeffizienten (robuste Standardfehler in Klammern; Koalitionen mit nur einer Partei ausgeschlossen):

$\begin{array}{lrr}\text { Heterogenität Landesparteien } & -0,30 & (0,15) \\ \text { Amtierende Regierung } & 4,08 & (0,67) \\ \text { Bundespolitik, R-Land } & 3,21 & (0,69) \\ \text { Bundespolitik, O-Land } & 3,07 & (0,59) \\ \text { Minderheitsregierung } & 0,62 & (1,03) \\ \text { Kleine Gewinnkoalition } & 1,01 & (0,87) \\ \text { Bargaining Proposition } & -0,48 & (0,73) \\ \text { Stärkste Partei } & 0,59 & (0,68),\end{array}$

wobei $\mathrm{N}=941$, Log-likelihood $=-74,95$, Pseudo- $\mathrm{R}^{2}=0,476$. Für ein ähnliches Verfahren des Einschlusses von Residuen vgl. Bueno de Mesquita et al. (2003: 137). 
Tabelle 3: Bedingte logistische Regression der Koalitionsbildung in den deutschen Bundesländern von 1990 bis 2007; ideologische Heterogenität nach Politikfeldern differenziert

\begin{tabular}{|c|c|c|c|c|}
\hline \multirow[t]{2}{*}{ Attribute } & \multicolumn{4}{|c|}{ Modelle } \\
\hline & (5) & (6) & (7) & (8) \\
\hline \multicolumn{5}{|l|}{ Office-Faktoren } \\
\hline Minderheitsregierung & $\begin{array}{l}-1,00 \\
(1,26)\end{array}$ & $\begin{array}{l}-0,21 \\
(1,50)\end{array}$ & $\begin{array}{l}-1,40 \\
(1,37)\end{array}$ & $\begin{array}{c}-1,74 \\
(1,43)\end{array}$ \\
\hline Kleine Gewinnkoalition & $\begin{array}{l}3,38 \\
(1,44)^{* *}\end{array}$ & $\begin{array}{l}4,32 \\
(1,56)^{* * *}\end{array}$ & $\begin{array}{l}4,05 \\
(1,50)^{* * *}\end{array}$ & $\begin{array}{l}3,26 \\
(1,47)^{* *}\end{array}$ \\
\hline Bargaining proposition & $\begin{array}{c}0,84 \\
(1,04)\end{array}$ & $\begin{array}{c}0,40 \\
(0,88)\end{array}$ & $\begin{array}{c}0,67 \\
(0,86)\end{array}$ & $\begin{array}{c}1,03 \\
(1,02)\end{array}$ \\
\hline Stärkste Partei & $\begin{array}{l}1,40 \\
(0,63)^{* *}\end{array}$ & $\begin{array}{l}1,53 \\
(0,64)^{* *}\end{array}$ & $\begin{array}{l}1,40 \\
(0,57)^{* *}\end{array}$ & $\begin{array}{l}1,07 \\
(0,66)\end{array}$ \\
\hline \multicolumn{5}{|l|}{ Policy-Faktoren } \\
\hline \multicolumn{5}{|l|}{ Heterogenität Landesparteien } \\
\hline Wirtschafts- und Sozialpolitik & $\begin{array}{l}-0,40 \\
(0,19)^{* *}\end{array}$ & $\begin{array}{l}-0,47 \\
(0,21)^{* *}\end{array}$ & $\begin{array}{l}-0,49 \\
(0,20)^{* *}\end{array}$ & \\
\hline Gesellschaftspolitik & $\begin{array}{c}0,01 \\
(0,14)\end{array}$ & $\begin{array}{c}0,02 \\
(0,13)\end{array}$ & $\begin{array}{c}0,03 \\
(0,13)\end{array}$ & \\
\hline \multicolumn{5}{|l|}{ Heterogenität Bundesparteien } \\
\hline Wirtschafts- und Sozialpolitik & & & & $\begin{array}{l}-0,34 \\
(0,14)^{* *}\end{array}$ \\
\hline Gesellschaftspolitik & & & & $\begin{array}{l}-0,18 \\
(0,12)\end{array}$ \\
\hline \multicolumn{5}{|l|}{ Kontextuelle Faktoren } \\
\hline Amtierende Regierung & $\begin{array}{l}1,99 \\
(0,51)^{* * *}\end{array}$ & $\begin{array}{l}1,29 \\
(0,58)^{* *}\end{array}$ & $\begin{array}{l}2,35 \\
(0,50)^{* * *}\end{array}$ & $\begin{array}{l}1,98 \\
(0,53)^{* * *}\end{array}$ \\
\hline \multicolumn{5}{|l|}{ Übereinstimmung mit Bundespolitik } \\
\hline R-Koalition & $\begin{array}{c}0,66 \\
(0,70)\end{array}$ & $\begin{array}{c}0,33 \\
(0,68)\end{array}$ & $\begin{array}{c}1,11 \\
(0,61)^{*}\end{array}$ & $\begin{array}{c}0,59 \\
(0,61)\end{array}$ \\
\hline O-Koalition & $\begin{array}{l}1,19 \\
(0,47)^{* *}\end{array}$ & $\begin{array}{c}0,58 \\
(0,55)\end{array}$ & $\begin{array}{c}0,97 \\
(0,64)\end{array}$ & $\begin{array}{c}0,67 \\
(0,58)\end{array}$ \\
\hline Vorwahlallianz & & $\begin{array}{l}2,39 \\
(1,01)^{* *}\end{array}$ & & \\
\hline Vorwahlallianz-Residuum & & & $\begin{array}{c}1,32 \\
(0,47)^{* * *}\end{array}$ & $\begin{array}{l}0,84 \\
(0,30)^{* * *}\end{array}$ \\
\hline $\mathrm{N}$ & 1215,00 & 1215,00 & 1215,00 & 1215,00 \\
\hline Pseudo $\mathrm{R}^{2}$ & 0,72 & 0,75 & 0,76 & 0,75 \\
\hline Log pseudolikelihood & $-50,82$ & $-46,28$ & $-43,86$ & $-45,73$ \\
\hline $\mathrm{AIC}$ & 119,64 & 112,56 & 107,72 & 111,46 \\
\hline $\mathrm{BIC}$ & 165,56 & 163,59 & 158,74 & 162,49 \\
\hline IIA-Test & 0,928 & 0,897 & 0,947 & 0,944 \\
\hline $\begin{array}{l}\text { Anteil korrekt vorhergesagter } \\
\text { Koalitionen }\end{array}$ & $75,4 \%$ & $75,4 \%$ & $78,3 \%$ & $75,4 \%$ \\
\hline
\end{tabular}

Anmerkungen: robuste Standardfehler in Klammern; * $\mathrm{p} \leq, 10 ; * * \mathrm{p} \leq, 05 ; * * * \mathrm{p} \leq, 01$. 
1,32 für die Übereinstimmung mit dem R- bzw. dem O-Länderlager auf Bundesebene). Die Ergebnisse lassen zum anderen auch den Schluss zu, dass die von den Parteien im Vorfeld von Wahlen geäußerten Koalitionspräferenzen einen eigenständigen Erklärungsfaktor darstellen, der sich nicht allein auf die übrigen Faktoren reduzieren lässt, die bei der späteren Regierungsbildung von Bedeutung sind. Schließlich legt ein Vergleich der Modelle 3 und 4 den Schluss nahe, dass die Heterogenität der Landesparteien die Koalitionsbildung besser abzubilden vermag als die Heterogenität der Bundesparteien zum jeweiligen Zeitpunkt. Kurz: Die programmatischen Haltungen der Landesparteien scheinen tatsächlich eine Rolle zu spielen.

Zur Untermauerung dieses Ergebnisses untersuchen wir weiterhin, welche Bedeutung der ideologischen Heterogenität jeweils auf den beiden in der Untersuchung berücksichtigten Politikfeldern „Wirtschaft und Soziales“ sowie „Gesellschaft“ zukommt. Die Ergebnisse sind in den Modellen 5 bis 8 in Tabelle 3 wiedergegeben. Die getrennte Betrachtung ermöglicht die Ermittlung der für die Koalitionsbildung auf Landesebene entscheidenden Policy-Dimension. Wie Tabelle 3 zeigt, hat der ermittelte Koeffizient für die gesellschaftspolitische Heterogenität in keinem der Modelle einen signifikanten Einfluss auf die Koalitionsbildung, während von der programmatischen Heterogenität auf der sozioökonomischen Dimension ein negativer Effekt ausgeht. Offenbar ist die Koalitionsbildung auf Landesebene vor allem vom wirtschaftspolitisch determinierten Links-Rechts-Gegensatz bestimmt und nicht von unterschiedlichen Auffassungen in gesellschaftspolitischen Fragen. Auch bei dieser Betrachtung der Koalitionsbildung zeigt sich, dass mit den Positionsdaten der Bundesparteien eine schlechtere Modellanpassung gelingt als mit den Positionsdaten der Landesparteien (Modell 8).

Abschließend soll noch näher untersucht werden, welche Effekte die Bundespolitik auf die Landespolitik bei der Regierungsbildung hat. Die obigen statistischen Analysen legen nahe, dass bestimmte Koalitionen gerade deshalb zustande kamen, weil sie zu den bundespolitischen Mehrheiten passten und so die Lager von entweder R-Ländern oder O-Ländern im Bundesrat stärken konnten. Alternative und aus anderen Gründen vielleicht präferierte Koalitionen wurden eventuell nicht eingegangen, weil sie als gemischte Länderregierungen weder dem Regierungs- noch dem Oppositionslager auf Bundesebene von Nutzen gewesen wären. Dazu führt Tabelle 4, ausgehend von Modell 3, diejenigen Situationen auf, bei denen die Abwesenheit des bundespolitischen Einflusses zu einer Veränderung der Koalitionsvorhersage führt. Es gibt insgesamt 15 Situationen, bei denen der bundespolitische Einfluss ausschlaggebend ist, wobei in acht dieser Konstellationen die Abwesenheit des Bundeseinflusses zur Veränderung einer korrekten Vorhersage führt. In vier Fällen wurde eine SPD/Grüne-Koalition prognostiziert, in zwei Situationen eine CDU/FDP- und in einer weiteren eine CDU/SPD-Koalition. Nach den Modellschätzungen wären hier ohne Bundeseinfluss Bündnisse aus SPD und FDP, aus CDU und PDS sowie eine Reihe Großer Koalitionen zustande gekommen. 
Tabelle 4: Situationen der Regierungsbildung mit ausschlaggebendem bundespolitischen Einfluss

\begin{tabular}{|c|c|c|c|}
\hline \multirow[t]{2}{*}{ Regierungsbildung } & \multirow[t]{2}{*}{ Tatsächliche Koalition } & \multicolumn{2}{|c|}{ Vorhergesagte Koalition } \\
\hline & & $\begin{array}{c}\text { mit } \\
\text { Bundeseinfluss }\end{array}$ & $\begin{array}{c}\text { ohne } \\
\text { Bundeseinfluss }\end{array}$ \\
\hline Berlin 1990 & $\mathrm{CDU} / \mathrm{SPD}$ & CDU/FDP & CDU/SPD \\
\hline Bremen 1991 & SPD/FDP/Grüne & SPD/Grüne & $\mathrm{SPD} / \mathrm{CDU}$ \\
\hline Bremen 2007 & SPD/Grüne & CDU/SPD & SPD/Grüne \\
\hline Hamburg 1993 & SPD/STATT & SPD/Grüne & $\mathrm{SPD} / \mathrm{CDU}$ \\
\hline Hamburg 1997* & SPD/Grüne & SPD/Grüne & $\mathrm{SPD} / \mathrm{CDU}$ \\
\hline Mecklenburg-Vorpommern 1990* & CDU/FDP & CDU/FDP & CDU/SPD \\
\hline Niedersachsen $1990^{*}$ & SPD/Grüne & SPD/Grüne & $\mathrm{SPD} / \mathrm{FDP}$ \\
\hline Nordrhein-Westfalen 1995* & SPD/Grüne & SPD/Grüne & $\mathrm{SPD} / \mathrm{CDU}$ \\
\hline Nordrhein-Westfalen 2000* & SPD/Grüne & SPD/Grüne & $\mathrm{SPD} / \mathrm{CDU}$ \\
\hline Rheinland-Pfalz 1991 & $\mathrm{SPD} / \mathrm{FDP}$ & SPD/Grüne & $\mathrm{SPD} / \mathrm{CDU}$ \\
\hline Sachsen 2004 & CDU/SPD & CDU/NPD & CDU/SPD \\
\hline Sachsen-Anhalt 1990* & CDU/FDP & CDU/FDP & CDU/SPD \\
\hline Sachsen-Anhalt 1998 & SPD & SPD/PDS & $\mathrm{SPD} / \mathrm{CDU}$ \\
\hline Sachsen-Anhalt 2006* & $\mathrm{CDU} / \mathrm{SPD}$ & CDU/SPD & $\mathrm{CDU} / \mathrm{PDS}$ \\
\hline Schleswig-Holstein 1996* & SPD/Grüne & SPD/Grüne & SPD/CDU \\
\hline
\end{tabular}

Anmerkung: vorhergesagte Koalitionen auf Basis der Schätzungen von Modell 3.

\section{Schlussbetrachtung}

In diesem Aufsatz sind wir der Frage nachgegangen, welche Faktoren die Regierungsbildung in den deutschen Bundesländern beeinflussen. Dazu wurde ein Datensatz verwendet, der neben gängigen Informationen wie dem Sitzanteil der Parteien im jeweiligen Landesparlament auch deren politikfeldspezifische programmatische Positionen, Salienzen und Koalitionspräferenzen beinhaltet. Die Ergebnisse der Analyse zeigen, dass nicht nur die parteipolitische Zusammensetzung von Regierung und Opposition auf Bundesebene einen Einfluss auf die Regierungsbildung in den Bundesländern hat, sondern dass auch die individuellen Präferenzen der Parteien „,vor Ort“ ins Gewicht fallen. Diese Präferenzen können sich im Wunsch nach der Fortsetzung der bestehenden Koalition äußern, in den Koalitionsaussagen der Parteien vor der Wahl zeigen oder sich an den programmatischen Standpunkten der Landesparteien orientieren. Wie die Beispiele aus Baden-Württemberg und Bremen gezeigt haben, nehmen die Landesparteien durchaus unterschiedliche Positionen ein, die sich noch dazu im Zeitverlauf ändern. Allerdings konnte auch nicht ausgeschlossen werden, dass die programmatischen Bewegungen der Parteien im Südwesten wie auch in der Hansestadt Bremen koalitionstaktisch motiviert waren.

Diese beiden zuletztgenannten Aspekte bieten Anreize für weitere Studien auf dem Gebiet des Parteienwettbewerbs im deutschen Mehrebenensystem. So kann erstens gefragt werden, inwiefern unterschiedliche Merkmale der Bundesländer wie beispielsweise deren ökonomisches Profil oder die sozialstrukturellen Eigenschaften der Wähler die programmatische Ausrichtung der Parteien beeinflussten. Des Weiteren stellen die pro- 
grammatischen Bewegungen der Landesparteien eine gute Ausgangslage für künftige Untersuchungen dar. So kann analysiert werden, inwiefern diese Veränderungen mit den Koalitionsaussagen im Zusammenhang stehen, die von den Parteien häufig getroffen werden und nicht unbedingt kongruent mit den Mustern des Parteienwettbewerbs auf Bundesebene sind.

Ein dritter Aspekt, der sich aus den Ergebnissen dieser Arbeit ergibt, ist vergleichender Natur. Spiegeln sich die für die deutschen Bundesländer gewonnenen Erkenntnisse in anderen Staaten, deren politisches System durch das Regieren in Mehrebenensystemen geprägt ist, wider? Anbieten würde sich hier eine Analyse des Parteienwettbewerbs in ,älteren“ Mehrebenensystemen wie der Schweiz, Österreich und Belgien auf der einen und ,jüngeren“ wie dem britischen und spanischen Modell auf der anderen Seite. Die vergleichende Studie von Downs (1998) bietet hierfür einen idealen Grundstock, den es mit weiterem Datenmaterial auszubauen gilt.

\section{Literatur}

Achen, Christopher H., 1986: The Statistical Analysis of Quasi-experiments. Berkeley: University of California Press.

Achen, Christopher H., 2000: Two-Step Hierarchical Estimation: Beyond Regression Analysis, in: Political Analysis 13, 447-456.

Alvarez, R. Michael/Glasgow, Garrett, 1999: Two-Stage Estimation of Nonrecursive Choice Models, in: Political Analysis 8, 147-165.

Alvarez, R. Michael/Nagler, Jonathan, 1998: When Politics and Models Collide: Estimating Models of Multiparty Elections, in: American Journal of Political Science 42, 55-96.

Andersen, Uwe/Woyke, Wichard (Hrsg.), 2003: Handwörterbuch des politischen Systems der Bundesrepublik Deutschland. Bonn: Bundeszentrale für politische Bildung.

Austen-Smith, David/Banks, Jeffrey, 1988: Elections, Coalitions, and Legislative Outcomes, in: American Political Science Review 82, 405-422.

Axelrod, Robert, 1970: Conflict of Interest. Chicago: Markham.

Bäck, Hanna/Dumont, Patrick, 2007: Combining Large-n and Small-n Strategies: The Way Forward in Coalition Research, in: West European Politics 30, 467-501.

Baron, David P./Ferejohn, John A., 1989: Bargaining in Legislatures, in: American Political Science Review 83, 1181-1206.

Baron, David P./Diermeier, Daniel, 2001: Elections, Governments, and Parliaments in Proportional Representation Systems, in: Quarterly Journal of Economics 116, 933-967.

Benoit, Kenneth/Laver, Michael, 2006: Party Policy in Modern Democracies. London: Routledge.

Bräuninger, Thomas/Ganghof, Steffen, 2005: Parteienwettbewerb im Zweikammersystem, in: Ganghof, Steffen/Manow, Philip (Hrsg.), Mechanismen der Politik. Strategische Interaktion im deutschen Regierungssystem. Frankfurt/New York: Campus Verlag, 149-181.

Bräuninger, Thomas/Debus, Marc, 2008: Parteienwettbewerb in den Bundesländern. [in Vorbereitung].

Bueno de Mesquita, Bruce/Smith, Alastair/Siverson, Randolph M./Morrow, James D., 2003: The Logic of Political Survival. Cambridge/Mass.: MIT Press.

Burkhart, Simone, 2005: Parteipolitikverflechtung. Über den Einfluss der Bundespolitik auf Landtagswahlentscheidungen von 1976 bis 2000, in: Politische Vierteljahresschrift 46, 14-38. 
Debus, Marc, 2007a: Die programmatische Entwicklung der deutschen Parteien auf Bundes- und Landesebene zwischen den Bundestagswahlen 1998 und 2005, in: Brettschneider, Frank/ Niedermayer, Oskar/Weßels, Bernhard (Hrsg.), Die Bundestagswahl 2005. Analysen des Wahlkampfs und der Wahlergebnisse. Wiesbaden: VS Verlag für Sozialwissenschaften, 43-63.

Debus, Marc, 2007b: Pre-Electoral Alliances, Coalition Rejections, and Multiparty Governments. Baden-Baden: Nomos.

Deutscher Bundestag, 2006: Gesetzentwurf der Fraktionen der CDU/CSU und SPD: Entwurf eines Gesetzes zur Änderung des Grundgesetzes (Artikel 22, 23, 33, 52, 72, 73, 74, 74a, 75, 84, 85, 87c, 91a, 91b, 93, 98, 104a, 104b, 105, 107, 109, 125a, 125b, 125c, 143c). Drucksache 16/813. Berlin.

Deschouwer, Kris, 2003: Political Parties in Multi-layered Systems, in: European Urban and Regional Studies 10, 213-226.

Diermeier, Daniel/Stevenson, Randolph T., 1999: Cabinet Survival and Competing Risks, in: American Journal of Political Science 43, 1051-1068.

Diermeier, Daniel/Eraslan, Hülya/Merlo, Antonio, 2003: A Structural Model of Government Formation, in: Econometrica 71, 27-70.

Dinkel, Rainer, 1977: Der Zusammenhang zwischen Bundes- und Landtagswahlergebnissen, in: Politische Vierteljahresschrift 18, 348-360.

Downs, William M., 1998: Coalition Government, Subnational Style: Multiparty Politics in Europe's Regional Parliaments. Columbus: Ohio State University Press.

Easton, David, 1965: A Framework for Political Analysis. Englewood Cliffs: Prentice Hall.

Fabritius, Georg, 1976: Der Bundesrat: Transmissionsriemen für die Unitarisierung der Bundesrepublik. Geschichte der Koalitionsbildung in den Bundesländern, in: Zeitschrift für Parlamentsfragen 7, 448-460.

Flick, Martina/Vatter, Adrian, 2007: Bestimmungsgründe der Parteienvielfalt in den deutschen Bundesländern, in: Politische Vierteljahresschrift 48, 44-65.

Freitag, Markus/Vatter, Adrian (Hrsg.), 2008: Die Demokratien der deutschen Bundesländer. Politische Institutionen im Vergleich. Opladen: Verlag Barbara Budrich.

Frankfurter Allgemeine Zeitung, 12. Mai 2007, 4.

Franklin, Mark N./Mackie, Thomas T., 1983: Familiarity and Inertia in the Formation of Governing Coalitions in Parliamentary Democracies, in: British Journal of Political Science 13, 275-298.

Golder, Sona, 2005: Pre-electoral Coalitions in Comparative Perspective: A Test of Existing Hypotheses, in: Electoral Studies 24, 643-663.

Hallermann, Andreas/Edinger, Michael, 2001: Rechtsextremismus in Ostdeutschland. Struktur und Ursachen rechtsextremer Einstellungen am Beispiel Thüringens, in: Zeitschrift für Parlamentsfragen 32, 588-612.

Hausman, Jerry/McFadden, Daniel, 1984: Specification Tests for the Multinomial Logit Model, in: Econometrica 52, 1219-1240.

Hinich, Melvin J./Munger, Michael C., 1997: Analytical Politics. Cambridge: Cambridge University Press.

Hough, Daniel/Jeffery, Charlie, 2003: Landtagswahlen: Bundestestwahlen oder Regionalwahlen?, in: Zeitschrift für Parlamentsfragen 34, 79-94.

Jun, Uwe, 1994: Koalitionsbildung in den deutschen Bundesländern. Theoretische Betrachtungen, Dokumentation und Analyse der Koalitionsbildungen auf Länderebene seit 1949. Opladen: Leske + Budrich.

Keele, Luke/Kelly, Nathan J., 2006: Dynamic Models for Dynamic Theories: The Ins and Outs of Lagged Dependent Variables, in: Political Analysis 14, 186-205.

King, Gary/Alt, James E./Burns, Nancy Elizabeth/Laver, Michael, 1990: A Unified Model of Cabinet Dissolution in Parliamentary Democracies, in: American Journal of Political Science 34, 846-871. 
Klein, Markus, 2005: Der Einfluss der beiden TV-Duelle im Vorfeld der Bundestagswahl 2002 auf die Wahlbeteiligung und die Wahlentscheidung Eine log-lineare Pfadanalyse auf der Grundlage von Paneldaten, in: Zeitschrift für Soziologie 34, 207-222.

König, Thomas, 2001: Bicameralism and Party Politics in Germany: An Empirical Social Choice Analysis, in: Political Studies 49, 411-437.

Kropp, Sabine, 2001: Regieren in Koalitionen. Handlungsmuster und Entscheidungsbildung in deutschen Länderregierungen. Wiesbaden: VS Verlag für Sozialwissenschaften.

Kropp, Sabine/Schüttemeyer, Suzanne S./Sturm, Roland, 2002: Koalitionen in West- und Osteuropa. Theoretische Überlegungen und Systematisierung des Vergleichs, in: Kropp, Sabine/ Schüttemeyer, Suzanne S./Sturm, Roland (Hrsg.), Koalitionen in West- und Osteuropa. Opladen: Leske + Budrich, 7-41.

Laver, Michael/Benoit, Kenneth/Garry, John, 2003: Extracting Policy Positions from Political Texts Using Words as Data, in: American Political Science Review 97, 311-331.

Laver, Michael/Hunt, W. Ben, 1992: Policy and Party Competition. New York, London: Routledge.

Laver, Michael/Schofield, Norman, 1998: Multiparty Government. The Politics of Coalition in Europe. Ann Arbor: University of Michigan Press.

Laver, Michael/Shepsle, Kenneth, 1996: Making and Breaking Governments. Cambridge/New York: Cambridge University Press.

Lehmbruch, Gerhard, 1976: Parteienwettbewerb im Bundesstaat. Regelsysteme und Spannungslagen im Institutionengefüge der Bundesrepublik Deutschland. Stuttgart: Kohlhammer.

Leiserson, Michael, 1968: Factions and Coalitions in One-Party Japan: An Interpretation Based on the Theory of Games, in: American Political Science Review 62, 770-787.

Lepsius, M. Rainer, 1973: Parteiensystem und Sozialstruktur, in: Ritter, Gerhard A. (Hrsg.), Die deutschen Parteien vor 1918. Köln: Vandenhoeck \& Ruprecht, 56-80.

Linhart, Eric, 2007: Rationales Wählen als Reaktion auf Koalitionssignale am Beispiel der Bundestagswahl 2005, in: Politische Vierteljahresschrift 48, 461-484.

Lipset, Seymour M./Rokkan, Stein, 1967: Cleavage Structures, Party Systems and Voter Alignments: An Introduction, in: Lipset, Seymour M./Rokkan, Stein (Hrsg.), Party Systems and Voter Alignments: Cross-National Perspectives. New York, London: Free Press, 1-64.

Martin, Lanny W./Stevenson, Randolph T., 2001: Government Formation in Parliamentary Democracies, in: American Journal of Political Science 45, 33-50.

McFadden, Daniel, 1974: Conditional Logit Analysis of Qualitative Choice Behaviour, in: Zarembka, Paul (Hrsg.), Frontiers in Econometrics. New York: Academic Press, 105-142.

Mielke, Siegfried/Reutter, Werner (Hrsg.), 2006: Länderparlamentarismus in Deutschland. Geschichte - Struktur - Funktion. Wiesbaden: VS Verlag für Sozialwissenschaften.

Pappi, Franz U., 1973: Parteiensystem und Sozialstruktur in der Bundesrepublik, in: Politische Vierteljahresschrift 14, 191-213.

Pappi, Franz U./Becker, Axel/Herzog, Alexander, 2005: Regierungsbildung in Mehrebenensystemen: Zur Erklärung der Koalitionsbildung in den deutschen Bundesländern, in: Politische Vierteljahresschrift 46, 432-458.

Pappi, Franz U./Gschwend, Thomas, 2005: Partei- und Koalitionspräferenzen der Wähler bei den Bundestagswahlen 1998 und 2002, in: Falter, Jürgen W./Gabriel, Oscar W./Weßels, Bernhard (Hrsg.), Wahlen und Wähler. Analysen aus Anlass der Bundestagswahl 2002. Wiesbaden: VS Verlag für Sozialwissenschaften, 284-305.

Pappi, Franz U./Herzog, Alexander/Schmitt, Ralf, 2006: Koalitionssignale und die Kombination von Erst- und Zweitstimmen bei den Bundestagswahlen 1953 bis 2005, in: Zeitschrift für Parlamentsfragen 37, 493-513.

Pappi, Franz U./Shikano, Susumu, 2004: Ideologische Signale in den Wahlprogrammen der deutschen Bundestagsparteien 1980 bis 2002, MZES Working Papers Nr. 76. Mannheim.

Powell, G. Bingham, 2000: Elections as Instruments of Democracy: Majoritarian and Proportional Visions. New Haven/London: Yale University Press. 
Rohe, Karl, 1992: Wahlen und Wählertraditionen in Deutschland. Kulturelle Grundlagen deutscher Parteien und Parteiensysteme im 19. und 20. Jahrhundert. Frankfurt a. M.: Suhrkamp.

Scherer, Michael, 2002: Freie Hansestadt Bremen, in: Wehling, Hans-Georg (Hrsg.), Die deutschen Länder. Geschichte, Politik, Wirtschaft. Opladen: Leske + Budrich, 109-120.

Scharpf, Fritz W., 1989: Decision Rules, Decision Styles, and Policy Choices, in: Journal of Theoretical Politics 1, 149-176.

Scharpf, Fritz W., 2000: Economic Changes, Vulnerabilities, and Institutional Capacities, in: Scharpf, Fritz W./Schmidt, Vivien A. (Hrsg.), Welfare and Work in the Open Economy, Band 2: From Vulnerability to Competitiveness. Oxford: Oxford University Press, 20-124.

Schmid, Josef, 1990: Die CDU. Organisationsstrukturen, Politiken und Funktionsweisen einer Partei im Föderalismus. Opladen: Leske + Budrich.

Schmidt, Manfred G., 2007: Das politische System Deutschlands. Institutionen, Willensbildung und Politikfelder. Bonn: Beck.

Schneider, Herbert, 2001: Parteien in der Landespolitik, in: Gabriel, Oscar W./Niedermayer, Oskar/Stöss, Richard (Hrsg.), Parteiendemokratie in Deutschland. Bonn: Bundeszentrale für politische Bildung, 385-405.

Sened, Itai, 1995: Equilibria in Weighted Voting Games with Sidepayments in Two Dimensional Spaces, in: Journal of Theoretical Politics 7, 283-300.

Sened, Itai, 1996: A Model of Coalition Formation: Theory and Evidence, in: Journal of Politics 58, 350-372.

Shikano, Susumu/Linhart, Eric, 2009: Coalition Formation as a Result of Policy and Office Motivations in the German Federal States: An Empirical Estimation of the Weighting Parameters of both Motivations, in: Party Politics 15 [im Erscheinen].

Strøm, Kaare, 1990a: A Behavioural Theory of Competitive Political Parties, in: American Journal of Political Science 34, 565-598.

Strøm, Kaare, 1990b: Minority Government and Majority Rule. Cambridge: Cambridge University Press.

Strøm, Kaare/Budge, Ian/Laver, Michael, 1994: Constraints on Cabinet Formation in Parliamentary Democracies, in: American Journal of Political Science 38, 303-335.

Sturm, Roland, 1996: Die baden-württembergische Landtagswahl vom 24. März 1996: Normalisierung oder kleinster gemeinsamer Nenner?, in: Zeitschrift für Parlamentsfragen 27, 602-616.

von Alemann, Ulrich/Brandenburg, Patrick, 2000: Nordrhein-Westfalen: Ein Land entdeckt sich neu. Köln: Kohlhammer.

von Neumann, John/Morgenstern, Oskar, 1944: Theory of Games and Economic Behaviour. Princeton: Princeton University Press.

Warwick, Paul, 1996: Coalition Government Membership in West European Parliamentary Democracies, in: British Journal of Political Science 46, 471-499.

Warwick, Paul, 2006: Policy Horizons and Parliamentary Government. Houndmills/Basingstoke: Palgrave Macmillan.

Wehling, Hans-Georg, 2002: Baden-Württemberg, in: Wehling, Hans-Georg (Hrsg.), Die deutschen Länder. Geschichte, Politik, Wirtschaft. Opladen: Leske + Budrich, 17-33.

Wehling, Hans-Georg, 2006: Föderalismus und politische Kultur in der Bundesrepublik Deutschland, in: Schneider, Herbert/Wehling, Hans-Georg (Hrsg.), Landespolitik in Deutschland. Grundlagen - Strukturen - Arbeitsfelder. Wiesbaden: VS Verlag für Sozialwissenschaften, 87-107.

Zohlnhöfer, Reimut, 1999: Die große Steuerreform 1998/99: Ein Lehrstück für Politikentwicklung bei Parteienwettbewerb im Bundesstaat, in: Zeitschrift für Parlamentsfragen 30, 326-345. 